\title{
In vitro Activity and Evaluation of Quality of Some Selected Penicillins on the Ghanaian Market using Developed HPLC Methods
}

\section{Rita Frema Boadu ${ }^{1}$, Christian Agyare ${ }^{1 *}$, Martin Adarkwa-Yiadom², Francis Adu ${ }^{1}$, Vivian Etsiapa Boamah ${ }^{1}$ and Yaw Duah Boakye ${ }^{1}$}

${ }^{1}$ Department of Pharmaceutics, Faculty of Pharmacy and Pharmaceutical Sciences, Kwame Nkrumah University of Science and Technology, Kumasi, Ghana ${ }^{2}$ Forensic Unit, Ghana Standard Authority, Accra, Ghana

\begin{abstract}
The use of antibiotics in health delivery is inevitable since it is one of the most prescribed medications. The quality and efficacy of these medications are crucial in health systems since they can affect the quality of healthcare delivery. The study was designed to determine the quality and activity of some penicillins on the Ghanaian market. A total of 54 samples (29 capsules and 25 suspensions) of different brands and batches were collected from different pharmacies in Accra and Kumasi, Ghana, from October 2011 to May 2012. The activity (zones of inhibition) and minimum inhibitory concentration (MIC) of the samples were determined by the agar-well diffusion and micro-dilution methods respectively against two typed strains of Gram-negative and Gram-positive bacteria. Quality of the samples was determined quantitatively by developed and validated HPLC methods. The MICs of flucloxacillin and cloxacillin samples were $\geq$ $1400 \mu \mathrm{g} / \mathrm{mL}$, whiles that of amoxicillin samples were $\geq 200 \mu \mathrm{g} / \mathrm{mL}$, with reference to the standard antibiotics which gave MICs of 200 to $800 \mu \mathrm{g} / \mathrm{mL}$ against all the test bacteria with the suspensions exhibiting higher antimicrobial activity. Specificity, linearity, precision and accuracy of the developed HPLC method were determined. HPLC analysis of the samples revealed that $75 \%$ of amoxicillin capsule samples and $92.3 \%$ of amoxicillin suspension samples contained the right amount of active pharmaceutical ingredient (API) with percentages ranging from 93.2 to $104.3 \%$ and 81.0 to $104.1 \%$ respectively. For samples of flucloxacillin capsules, $62.5 \%$ of the samples showed API content from 96 to $120.5 \%$. All the suspension samples have their API within BP and USP specification of 114.4 to $120.0 \%$. Capsules $(58.6 \%)$ of all the samples contained the right API whereas $64 \%$ of them were recorded for suspensions. Out of the 54 samples evaluated, $61.1 \%$ were within the BP and USP specifications. The biological assay revealed higher MIC values for all the penicillin samples evaluated compared with the reference samples. Among the samples evaluated, amoxicillin showed better quality of $82.8 \%$ as compared to flucloxacillin $(31.3 \%)$ and cloxacillin $(44.4 \%)$ samples. Efforts should therefore be made to improve the quality and storage conditions of these antibiotics and also constant monitoring and surveillance of activity and potency of these antibiotics should be done.
\end{abstract}

Keywords: Penicillins; HPLC; Minimum inhibitory concentration (MIC); Active pharmaceutical ingredient (API)

\section{Introduction}

The World Health Organization (WHO) defines counterfeit products as those which are deliberately and fraudulently mislabeled with respect to identity or source $[1,2]$. Substandard medicines, on the other hand, are medicines that do not meet official standards and specification for strength, quality, purity, packaging, and labeling and their presence are one of the latest threats facing the pharmaceutical industry and healthcare delivery system globally. As a result of weak or no regulatory systems in many low and middle income countries [3,4], most of the medicines in circulation in these countries do not meet internationally accepted quality and specification and may be detrimental to patients.

The total worldwide trade in counterfeit medicines is estimated to be 5 to $7 \%$ of the pharmaceutical market [5]. The problem is more severe in developing countries. More than $30 \%$ of all medicines sold in Africa are counterfeit medicines [6]. Counterfeit and substandard medicines are not only available in the developing countries but also in the developed world [7]. In 1999, 22\% of the 771 reports of counterfeited medicines received by WHO came from the developed countries, the remaining $78 \%$ were from the developing countries [3].

Prevalence of counterfeit and substandard medicines has a major effect on the health delivery system. They can result in treatment failure, toxicity, adverse reaction or severe side effects thereby increasing mortality rate [8]. Counterfeit and substandard medicines may be found in all classes of medicines. The two major classes most counterfeited in the developing countries are anti-parasitic and anti-infective medicines [2]. Exposure of microorganisms to counterfeit and substandard antiinfectives leads to antimicrobial resistance, thereby putting health of patients at risk [9]. Antimicrobial resistance contributes to high cost of healthcare as patients using these counterfeit and substandard medicines do not respond to treatment and have to resort to higher doses and newer medicines. Additionally, patients remain ill for longer period leading to the loss of productivity $[1,10]$. Infectious diseases are taking lives of people and believed to be the world's leading cause of death. It is estimated that 50,000 people die a day out of infectious diseases [11].

Medicines need to be of acceptable quality, safety and efficacy, especially antibiotics [12]. The appropriate active pharmaceutical ingredients (API) quantity and its efficacy to effect treatment must be ascertained. This is achieved through analysis and comparison to the manufacturer's specifications or standard specification in the pharmacopoeias. Consequently, there is the need to sample and evaluate some of the antibiotics on the Ghanaian market to ensure that they meet the required specifications as spelt out in the United States Pharmacopoeia (USP) and British Pharmacopoeia (BP) to avoid all the problems associated with counterfeit and substandard medicines.

*Corresponding author: Dr. Christian Agyare, Department of Pharmaceutics, Faculty of Pharmacy and Pharmaceutical Sciences, Kwame Nkrumah University of Science and Technology, Kumasi, Ghana, Tel: +233246369803 E-mail: cagyare.pharm@knust.edu.gh; chrisagyare@yahoo.com

Received December 01, 2014; Accepted January 09, 2015; Published January 12, 2015

Citation: Boadu RF, Agyare C, Yiadom MA, Adu F, Boamah VE, et al. (2015) In vitro Activity and Evaluation of Quality of Some Selected Penicillins on the Ghanaian Market using Developed HPLC Methods. Med chem 5: 001-014. doi:10.4172/2161-0444.1000235

Copyright: $\odot 2015$ Boadu RF, et al. This is an open-access article distributed under the terms of the Creative Commons Attribution License, which permits unrestricted use, distribution, and reproduction in any medium, provided the original author and source are credited. 
Antibiotics are natural or synthetic chemical agents that can inhibit the growth or kill microorganisms [13]. Antibiotics are one class of antimicrobials and they are either referred to as bactericidal or bacteriostatic when they kill or inhibit growth or bacteria respectively [14]. They are heterogeneous and the only common property is that they are all organic in nature. A required feature of any antibiotic is its effect on bacteria at low concentration since that differentiate antibiotics from other compounds which have antimicrobial effect at higher concentrations e.g. ethanol. The discovery of antibiotics have significantly reduced mortality resulting from infectious diseases and also facilitated the success rates of many medical procedures such as surgery $[15,16]$. They are also employed extensively to prevent and treat infectious diseases in humans and animals [17]. These agents are mostly directed against some targets that are peculiar to bacteria, interfering with the growth of sensitive structures or processes that are critical to the survival and growth of the bacteria. Antibiotics inhibit sensitive bacteria by blocking important macromolecules like enzymes and nucleic acid activity which are very important in cell multiplication or division [18]. In effect, they are able to bind to specific site on the macromolecule to form a complex, different from the original entity and are unable to perform its function. The main targets are bacterial cell wall synthesis (peptidoglycan), bacterial protein synthesis (bacterial ribosome), bacterial DNA replication (bacterial enzymes involved in DNA supercoiling) and cytoplasmic membrane function [19]. The aim of this study was to determine the antibacterial activity and develop HPLC methods to analyze API content of various samples of amoxicillin, flucloxacillin and cloxacillin on the Ghanaian market.

\section{Materials}

\section{Chemicals and reference drugs}

All chemicals used for the HPLC analysis including reference compounds such as amoxicillin trihydtrate (96\% HPLC), flucloxacillin (98\% HPLC), cloxacillin (98\% HPLC), caffeine anhydrous (98\% HPLC) and acetaminophen (98\% HPLC), solvents etc. were of analytical and chromatographic grade purchased from Sigma-Aldrich, Darmstadt, Germany unless otherwise stated and they were available in the Forensic Laboratory of Ghana Standard Authority, Accra, Ghana. All materials and equipment used in the microbiological evaluation are available in the Microbiology Section, Department of Pharmaceutics, Kwame Nkrumah University of Science and Technology (KNUST), Kumasi, Ghana.

\section{Test bacteria}

Four typed strains of bacteria consisting of two Gram-negative and two Gram-positive bacteria were used for the microbiological evaluation. All organisms were typed cultures stored at the Microbiology Research Laboratory, Department of Pharmaceutics, KNUST, Kumasi, Ghana with the following identities: Escherichia coli ATCC 25922, Pseudomonas aeruginosa ATCC 4853, Staphyloccocus aureus ATCC 25923 and Bacillus subtilis NTCC 10073.

\section{Test penicillin samples}

Imported and locally manufactured penicillin samples were randomly purchased from different Pharmacies in Accra and Kumasi, Ghana. The reasons for the choice of samples were to compare different brands and different batches within a brand. Sampling of antibiotics was done from October, 2011 to May, 2012.

\section{Methods}

\section{Determination of antibacterial activity}

The antimicrobial activity was determined using modified method described by Agyare et al. [20] and Girish and Satish [21]. Twenty (20) milliliters stabilized agar at $45^{\circ} \mathrm{C}$ was seeded with $100 \mu \mathrm{L}$ of $10^{5}$ colony forming units ( $\mathrm{cfu}) / \mathrm{mL}$ of 18 to $24 \mathrm{~h}$ broth culture of $S$. aureus and rolled in the palm for uniform distribution and was aseptically poured into sterilized Petri dish and allowed to set. Four wells were bored with diameter of $10 \mathrm{~mm}$. The wells were filled with $200 \mu \mathrm{L}$ each of respective concentrations and allowed to stand for $1 \mathrm{~h}$ on the bench to allow diffusion of antibiotic. The plate was then incubated at $37^{\circ} \mathrm{C}$ for $24 \mathrm{~h}$ and zones of growth inhibition recorded in millimeter ( $\mathrm{mm}$ ). The method used was performed in triplicate for all test samples using B. subtilis, E. coli and P. aeruginosa. Concentrations used were 0.125 to $1.0 \mu \mathrm{g} / \mathrm{mL}$ for amoxicillin samples and 1.25 to $10.0 \mathrm{mg} / \mathrm{mL}$ for flucloxacillin and cloxacillin samples.

\section{Determination of minimum inhibitory concentration}

Minimum inhibitory concentrations (MIC) of the various antibiotic samples were determined using the method described by Agyare et al. [20]. Sterile 96-well microtitre plates were labeled appropriately for $S$. aureus. Total volume of $200 \mu \mathrm{L}$ were prepared by dispensing a fixed volume of $100 \mu \mathrm{L}$ sterile double strength nutrient broth and $20 \mu \mathrm{L}\left(10^{5} \mathrm{cfu} / \mathrm{mL}\right)$ of $18 \mathrm{~h}$ culture was aseptically added to the medium. Amoxicillin samples were evaluated within concentration range of 0.1 to $0.5 \mathrm{mg} / \mathrm{mL}$. The MIC of flucloxacillin and cloxacillin samples was determined within a concentration range of 0.5 to 2.2 $\mathrm{mg} / \mathrm{mL}$. Experiments were performed in triplicate under the same conditions for all samples. Reference samples were prepared and the MIC determined under the same conditions as described above.

The plates were incubated at $37^{\circ} \mathrm{C}$ for $24 \mathrm{~h}$. Microbial growth was determined by addition of $30 \mu \mathrm{L}$ 3-(4,5-dimethylthiazole -2-yl)-2,5diphenyltetrazolium bromide (MTT) after incubation and as growth of organism was indicated by purple to blue coloration and yellow coloration indicated no growth of organism. The well with least concentration of test sample without bacterial growth recorded as the MIC. The procedure above was repeated for all test samples using $E$. coli, B. subtilis and P. aeruginosa respectively.

\section{HPLC analysis of reference and test samples}

Reference amoxicillin trihydrate samples were dissolved in $0.1 \mathrm{M}$ hydrochloric acid. Samples were analyzed at concentrations of 5.26, $10.52,15.78,21.04$ and $26.3 \mu \mathrm{g} / \mathrm{mL}$ with an injection volume of 100 $\mu \mathrm{L}$. Reference flucloxacillin and cloxacillin samples were dissolved in sterile distilled millipore water. They were analyzed at concentrations of $25.35,50.7,101.4,152.1 \mu \mathrm{g} / \mathrm{mL}$ and $11.72,23.44,35.16,58.6 \mu \mathrm{g} / \mathrm{mL}$ for reference standard and the sample respectively, with an injection volume of $1 \mathrm{~mL}$. All samples were analyzed under isocratic conditions with Shim-Pac CLS ODS (M) C18 column for amoxicillin. ShimPac CLC- $\mathrm{NH}_{2}$ C18 column was used in analysis of flucloxacillin and cloxacillin. An internal standard of $1025 \mu \mathrm{g} / \mathrm{mL}$ caffeine anhydrous was used in the development of HPLC method for amoxicillin and analysis of amoxicillin trihydrate samples. Concentrations of $1.4156 \mu \mathrm{M}$ and $1.3296 \mu \mathrm{M}$ of acetaminophen (paracetamol) were used for the HPLC method development for flucloxacillin and cloxacillin respectively. The same concentrations were used for the analysis of flucloxacillin and cloxacillin samples.

\section{Preparation of test sample solutions}

Concentrations of amoxicillin trihydrate equivalent to $15.78 \mu \mathrm{g} /$ $\mathrm{mL}$ were prepared. They were dissolved in $0.1 \mathrm{M}$ hydrochloric acid and mobile phase consisting of methanol/ $0.01 \mathrm{M}$ potassium dihydrogen phosphate $(65: 35, \mathrm{v} / \mathrm{v})$. Equivalent of 50.7 and $11.72 \mu \mathrm{g} / \mathrm{mL}$ of flucloxacillin and cloxacillin were prepared. Samples were dissolved in sterile distilled water and mobile phase. 


\section{Statistical analysis}

All graphs were plotted with Excel version 2010 and graph pad prism (Graph Pad Prism 5 Software, San Diego, CA, USA) for all the statistical analysis. Data analysis was by one-way analysis of variance (ANOVA). There is not enough evidence at alpha $=0.05$ and the model for the method development is not significant since F-value $>$ F-crit and $\mathrm{P}<0.05$ (alpha). ChromQuest and Endnote X6 (Bld 6348) were used to generate HPLC analysis data and references respectively.

\section{Results}

\section{Antibacterial activities of samples}

The MICs of capsules were within the range of 200 to $800 \mu \mathrm{g} / \mathrm{mL}$ for amoxicillin trihydrate samples and $\geq 800$ to 1900 for flucloxacillin and cloxacillin test samples. Reference amoxicillin samples showed lower MICs of $200 \mu \mathrm{g} / \mathrm{mL}$ against E. coli, $500 \mu \mathrm{g} / \mathrm{mL}$ against $P$. aeruginosa, $300 \mu \mathrm{g} / \mathrm{mL}$ against $B$. subtilis and $200 \mu \mathrm{g} / \mathrm{mL}$ against $S$. aureus. MICs of reference flucloxacillin sample were $800 \mu \mathrm{g} / \mathrm{mL}$ against $E$. coli, $1500 \mu \mathrm{g} /$ $\mathrm{mL}$ for P. aeruginosa, $1400 \mu \mathrm{g} / \mathrm{mL}$ for B. subtilis and $1400 \mu \mathrm{g} / \mathrm{mL}$ for $S$. aureus. MICs for reference cloxacillin sample were $800 \mu \mathrm{g} / \mathrm{mL}$ against E. coli, $1500 \mu \mathrm{g} / \mathrm{mL}$ against $P$. aeruginosa, $1500 \mu \mathrm{g} / \mathrm{mL}$ against $B$. subtilis and $1500 \mu \mathrm{g} / \mathrm{mL}$ for S. aureus (Table 1).

Antibacterial activity of sampled antibiotic suspensions of amoxicillin, flucloxacillin and cloxacillin samples. Evaluation of samples gave MICs within the range of 200 to $700 \mu \mathrm{g} / \mathrm{mL}$ for amoxicillin test samples, 800 to 1600 for flucloxacillin and 500 to 1700 cloxacillin samples (Table 2).

Antibacterial activity of sampled antibiotic capsules of amoxicillin, flucloxacillin and cloxacillin samples. Evaluation of samples at test concentrations gave mean zones of inhibition within the range of 0.0 to $30.0 \mathrm{~mm}$ for amoxicillin test samples, 0.0 to $31.67 \mathrm{~mm}$ for flucloxacillin and 0.00 to $29.83 \mathrm{~mm}$ for cloxacillin samples (Table 3).

Antibacterial activity of sampled antibiotic suspensions of amoxicillin, flucloxacillin and cloxacillin samples. Evaluation of samples at test concentrations gave mean zones of inhibition within the range of 0.0 to $28.67 \mathrm{~mm}$ for amoxicillin test samples, 0.0 to $37.83 \mathrm{~mm}$ for flucloxacillin and 0.0 to $33.83 \mathrm{~mm}$ for cloxacillin samples (Table 4).

Antibacterial activity of reference antibiotic of amoxicillin, flucloxacillin and cloxacillin samples. Evaluation of samples at test concentrations gave mean zones of inhibition within the range of 0.00 to $30.83 \mathrm{~mm}$ for amoxicillin test samples, 0.00 to $38.00 \mathrm{~mm}$ for flucloxacillin and 0.00 to $30.00 \mathrm{~mm}$ for cloxacillin samples (Table 5).

\section{HPLC analysis of amoxicillin samples}

The active pharmaceutical ingredients (APIs) in the samples were determined using the developed and validated HPLC method. The chromatographic conditions for the analysis of amoxicillin trihydrate were made up of mobile phase consisting of methanol: $0.01 \mathrm{M}$ potassium dihydrogen phosphate $(65: 35, \mathrm{v} / \mathrm{v})$ yielded maximum sensitivity and separation. Flow rates between 0.5 and $1.2 \mathrm{~mL} / \mathrm{min}$ on a Shim-pack CLS-ODS C18 (M) $250 \times 4.6 \mathrm{~mm}, 5$ microns column were studied and a flow rate of $1.0 \mathrm{~mL} / \mathrm{min}$ gave an optimal signal to noise ratio with a reasonable separation time of $1.42 \mathrm{~min}$ for amoxicillin when injected alone.

HPLC chromatogram of amoxicillin (Figure 1) as reference sample alone and reference amoxicillin and caffeine as internal standard (Figure 2). The running time of the reference sample and the internal standard was less than $3 \mathrm{~min}$. The major peak at $1.421 \mathrm{~min}$ is for amoxicillin whereas that for caffeine is $2.974 \mathrm{~min}$ (Figure 1).
A five-point calibration curve was generated for amoxicillin in the concentration range of 5.26 to $263.0 \mu \mathrm{g} / \mathrm{mL}$ (Figure 3). The calibration curve provided a linear relationship between the peak area (y-axis) and the concentrations of amoxicillin trihydrate with the regression equation of $\mathrm{y}=194.41 \mathrm{x}+0.004, \mathrm{R}^{2}=0.9995$ (Figure 3 ). The residual points of the calibration curve were well distributed within acceptable limits (Figure 4).

Regression analysis cannot minimize the distance for all points simultaneously but does it for most of the points. The residual plot of points shows maximum points closer to line for amoxicillin (Figure 4).

The developed HPLC methods were validated using the International Conference on Harmonization guidelines and the parameters therein. It was performed using a well-designed experiment and statistically relevant methods in accordance with International Conference on Harmonization (ICH) guidelines on validation of analytical procedures $[22,23]$.

The linearity of the detector response for amoxicillin was confirmed from 5.26 to $263.0 \mu \mathrm{g} / \mathrm{mL}$. The calibration curve (Figure 3) and the residuals (Figure 4) were inspected to asses linearity (Table 6).

Table 1: MICs of capsule samples of amoxicillin, flucloxacillin and cloxacillin.

\begin{tabular}{|c|c|c|c|c|}
\hline \multirow[t]{2}{*}{ Sample } & \multicolumn{4}{|c|}{ Organisms/MIC ( $\mu \mathrm{g} / \mathrm{mL})$} \\
\hline & E. coli & P. aeruginosa & B. subtilis & S. aureus \\
\hline & & AMOXICILLIN & & \\
\hline $\begin{array}{c}\text { Reference } \\
\text { sample }\end{array}$ & 200 & 500 & 300 & 200 \\
\hline $01 \mathrm{~A}$ & 200 & 500 & 400 & 400 \\
\hline 01B & 300 & 700 & 500 & 500 \\
\hline $02 \mathrm{~A}$ & 300 & 700 & 400 & 200 \\
\hline 02B & 400 & 800 & 400 & 300 \\
\hline 03A & 200 & 600 & 300 & 300 \\
\hline 03B & 200 & 700 & 300 & 300 \\
\hline $03 \mathrm{C}$ & 200 & 600 & 300 & 300 \\
\hline $04 \mathrm{~A}$ & 200 & 700 & 300 & 300 \\
\hline $05 \mathrm{~A}$ & 300 & 600 & 300 & 300 \\
\hline $06 \mathrm{~A}$ & 400 & 800 & 400 & 400 \\
\hline 06B & 400 & 700 & 500 & 300 \\
\hline $06 \mathrm{C}$ & 300 & 700 & 500 & 400 \\
\hline 07A & 200 & 500 & 300 & 200 \\
\hline 07B & 400 & 800 & 400 & 400 \\
\hline $08 \mathrm{~A}$ & 300 & 700 & 400 & 400 \\
\hline \multirow[t]{2}{*}{ 09A } & 300 & 500 & 300 & 200 \\
\hline & & FLUCLOXACILLIN & & \\
\hline Reference & 800 & 1500 & 1400 & 1400 \\
\hline FLMG01 & 1300 & 1900 & 1500 & 1500 \\
\hline FLMG02 & 1200 & 1700 & 1400 & 1500 \\
\hline FLMG02 & 800 & 1500 & 1500 & 1500 \\
\hline FLLP04 & 1300 & 1800 & 1500 & 1500 \\
\hline FLLP05 & 1200 & 1600 & 1500 & 1500 \\
\hline FLLP06 & 1300 & 1700 & 1500 & 1500 \\
\hline FLAR07 & 800 & 1600 & 1500 & 1500 \\
\hline \multirow[t]{2}{*}{ FLAR08 } & 800 & 1600 & 1500 & 1500 \\
\hline & & CLOXACILLIN & & \\
\hline Reference & 800 & 1500 & 1500 & 1500 \\
\hline CLLP01 & 800 & 1500 & 1500 & 1500 \\
\hline CLLP02 & 900 & 1600 & 1500 & 1500 \\
\hline CLLP03 & 800 & 1500 & 1500 & 1500 \\
\hline CLAR04 & 900 & 1600 & 1500 & 1500 \\
\hline CLAR05 & 800 & 1500 & 1500 & 1500 \\
\hline CLMG06 & 800 & 1400 & 1500 & 1400 \\
\hline
\end{tabular}

$\mathrm{MIC}=$ minimum inhibitory concentration $(\mu \mathrm{g} / \mathrm{mL})$ 
Citation: Boadu RF, Agyare C, Yiadom MA, Adu F, Boamah VE, et al. (2015) In vitro Activity and Evaluation of Quality of Some Selected Penicillins on the Ghanaian Market using Developed HPLC Methods. Med chem 5: 001-014. doi:10.4172/2161-0444.1000235

\begin{tabular}{|c|c|c|c|c|}
\hline \multirow{2}{*}{ Sample } & \multicolumn{4}{|c|}{ Organisms/MIC $(\mu \mathrm{g} / \mathrm{mL})$} \\
\hline & E. coli & P. aeruginosa & B. subtilis & S. aureus \\
\hline & & AMOXICILLIN & & \\
\hline S01 & 300 & 600 & 300 & 200 \\
\hline $\mathrm{S} 02 \mathrm{~A}$ & 200 & 500 & 300 & 200 \\
\hline S02B & 300 & 500 & 300 & 200 \\
\hline $\mathrm{S} 02 \mathrm{C}$ & 300 & 600 & 300 & 200 \\
\hline S03A & 200 & 500 & 300 & 200 \\
\hline S04A & 300 & 600 & 300 & 200 \\
\hline S05A & 300 & 500 & 300 & 200 \\
\hline S06A & 200 & 500 & 300 & 200 \\
\hline S06B & 300 & 700 & 400 & 300 \\
\hline S06C & 300 & 600 & 300 & 300 \\
\hline S07A & 200 & 500 & 300 & 200 \\
\hline S08A & 200 & 500 & 300 & 200 \\
\hline \multirow[t]{2}{*}{ S08B } & 200 & 500 & 300 & 200 \\
\hline & & FLUCLOXACILLIN & & \\
\hline FLSMG01 & 800 & 1500 & 1400 & 1400 \\
\hline FLSMG02 & 800 & 1600 & 1400 & 1400 \\
\hline FLSMG03 & 800 & 1500 & 1400 & 1500 \\
\hline FLSLP04 & 800 & 1600 & 1400 & 1600 \\
\hline FLSLP05 & 800 & 1600 & 1600 & 1600 \\
\hline FLSLP06 & 800 & 1500 & 1500 & 1400 \\
\hline FLSAR07 & 800 & 1500 & 1400 & 1400 \\
\hline \multirow[t]{2}{*}{ FLSAR08 } & 800 & 1500 & 1600 & 1400 \\
\hline & & CLOXACILLIN & & \\
\hline CLSLP01 & 800 & 1500 & 1500 & 1600 \\
\hline CLSLP02 & 800 & 1700 & 1500 & 1500 \\
\hline CLSLP03 & 800 & 1600 & 500 & 1500 \\
\hline CLSMG04 & 800 & 1500 & 1600 & 1600 \\
\hline CLSMG05 & 800 & 1600 & 1600 & 1600 \\
\hline
\end{tabular}

Table 3: Antibacterial activity (mean zones of inhibition \pm SEM) of test samples (capsules).

\begin{tabular}{|c|c|c|c|c|c|}
\hline \multirow[b]{2}{*}{ Samples } & \multicolumn{5}{|c|}{ Organisms } \\
\hline & $\begin{array}{c}\text { Concentrations } \\
(\mu \mathrm{g} / \mathrm{mL})\end{array}$ & S. aureus & E. coli & B. subtilis & $\begin{array}{c}P . \\
\text { aeruginosa }\end{array}$ \\
\hline \multicolumn{6}{|c|}{ AMOXICILLIN } \\
\hline \multirow{4}{*}{$01 \mathrm{~A}$} & 1000 & $22.33 \pm 0.82$ & $16.00 \pm 0.63$ & $20.50 \pm 0.55$ & $21.67 \pm 0.52$ \\
\hline & 500 & $20.83 \pm 0.75$ & $12.67 \pm 0.52$ & $18.50 \pm 0.55$ & $19.33 \pm 0.52$ \\
\hline & 250 & $25.00 \pm 0.0$ & $12.00 \pm 0.0$ & $18.17 \pm 0.41$ & $17.83 \pm 0.75$ \\
\hline & 125 & 0.0 & 0.0 & 0.0 & 0.0 \\
\hline \multirow{4}{*}{ 01B } & 1000 & $25.83 \pm 0.41$ & $26.66 \pm 0.52$ & $24.67 \pm 0.82$ & $21.67 \pm 0.52$ \\
\hline & 500 & $25.00 \pm 0.63$ & $24.67 \pm 0.82$ & $23.00 \pm 0.63$ & $19.67 \pm 0.82$ \\
\hline & 250 & $22.67 \pm 0.52$ & $22.67 \pm 0.52$ & $21.00 \pm 0.89$ & $18.33 \pm 1.37$ \\
\hline & 125 & 0.0 & 0.0 & 0.0 & 0.0 \\
\hline \multirow{4}{*}{$02 \mathrm{~A}$} & 1000 & $25.67 \pm 1.03$ & $24.00 \pm 0.9$ & $19.00 \pm 00$ & $23.50 \pm 0.55$ \\
\hline & 500 & $23.33 \pm 1.03$ & $17.50 \pm 0.55$ & $14.17 \pm 0.75$ & $22.50 \pm 0.84$ \\
\hline & 250 & $22.17 \pm 0.41$ & $16.17 \pm 0.75$ & $17.00 \pm 0.0$ & $21.50 \pm 0.55$ \\
\hline & 125 & 0.0 & 0.0 & 0.0 & 0.0 \\
\hline \multirow{4}{*}{ 02B } & 1000 & $25.33 \pm 0.52$ & $23.33 \pm 1.21$ & $25.33 \pm 0.51$ & $23.00 \pm 0.89$ \\
\hline & 500 & $24.50 \pm 1.38$ & $22.50 \pm 0.55$ & $24.83 \pm 0.98$ & $20.83 \pm 1.17$ \\
\hline & 250 & $22.50 \pm 1.05$ & $18.50 \pm 1.05$ & $22.67 \pm 0.52$ & $18.50 \pm 0.84$ \\
\hline & 125 & 0.0 & 0.0 & 0.0 & 0.0 \\
\hline \multirow{4}{*}{$03 \mathrm{~A}$} & 1000 & $24.8 . \pm 0.41$ & $20.83 \pm 0.52$ & $24.50 \pm 0.84$ & 0.0 \\
\hline & 500 & $21.83 \pm 0.41$ & $23.83 \pm 0.75$ & $24.00 \pm 0.89$ & 0.0 \\
\hline & 250 & $20.83 \pm 0.41$ & $18.83 \pm 0.75$ & $22.50 \pm 0.84$ & 0.0 \\
\hline & 125 & 0.0 & 0.0 & 0.0 & 0.0 \\
\hline \multirow{4}{*}{ 03B } & 1000 & $25.83 \pm 0.98$ & $20.83 \pm 0.75$ & $24.83 \pm 0.75$ & $20.67 \pm 1.03$ \\
\hline & 500 & $22.67 \pm 1.21$ & $18.00 \pm 0.63$ & $23.83 \pm 0.41$ & $17.83 \pm 0.75$ \\
\hline & 250 & $21.17 \pm 0.98$ & $12.67 \pm 0.52$ & $20.67 \pm 0.82$ & $16.33 \pm 0.82$ \\
\hline & 125 & 0.0 & 0.0 & 0.0 & 0.0 \\
\hline $03 \mathrm{C}$ & 1000 & $24.67 \pm 1.00$ & $18.67 \pm 0.52$ & $23.50 \pm 0.55$ & 0.0 \\
\hline
\end{tabular}

\begin{tabular}{|c|c|c|c|c|c|}
\hline & 500 & $22.17 \pm 1.17$ & $16.83 \pm 0.98$ & $21.33 \pm 1.37$ & 0.0 \\
\hline & 250 & $20.67 \pm 1.21$ & $15.00 \pm 0.89$ & $20.50 \pm 1.38$ & 0.0 \\
\hline & 125 & 0.0 & 0.0 & 0.0 & 0.0 \\
\hline \multirow{4}{*}{$04 \mathrm{~A}$} & 1000 & $26.57 \pm 1.05$ & $20.71 \pm 0.36$ & $23.86 \pm 0.75$ & $0.00 \pm 0.00$ \\
\hline & 500 & $22.14 \pm 0.84$ & $18.43 \pm 0.52$ & $21.57 \pm 0.52$ & 0.0 \\
\hline & 250 & $21.43 \pm 1.21$ & $22.50 \pm 0.71$ & $20.29 \pm 0.82$ & 0.0 \\
\hline & 125 & 0.0 & 0.0 & 0.0 & 0.0 \\
\hline \multirow{4}{*}{ 05A } & 1000 & $30.00 \pm 0.89$ & $23.00 \pm 0.0$ & $24.50 \pm 0.84$ & 0.0 \\
\hline & 500 & $27.67 \pm 1.03$ & $26.00 \pm 0.0$ & $21.33 \pm 1.03$ & 0.0 \\
\hline & 250 & $25.67 \pm 1.03$ & $24.00 \pm 0.0$ & $20.17 \pm 0.98$ & 0.0 \\
\hline & 125 & 0.0 & 0.0 & 0.0 & 0.0 \\
\hline \multirow[t]{4}{*}{$06 \mathrm{~A}$} & 1000 & $21.00 \pm 0.0$ & $18.87 \pm 0.4$ & $22.83 \pm 0.14$ & $22.00 \pm 0.00$ \\
\hline & 500 & $20.00 \pm 0.0$ & $22.00 \pm 0.0$ & $22.30 \pm 0.18$ & $21.67 \pm 0.18$ \\
\hline & 250 & $18.00 \pm 0.0$ & $21.00 \pm 0.17$ & $21.00 \pm 0.18$ & $20.00 \pm 0.0$ \\
\hline & 125 & 0.0 & 0.0 & 0.0 & 0.0 \\
\hline \multirow{4}{*}{ 06B } & 1000 & $25.50 \pm 0.55$ & $15.50 \pm 0.84$ & $16.00 \pm 0.82$ & 0.0 \\
\hline & 500 & $24.50 \pm 0.84$ & $12.67 \pm 0.52$ & $12.00 \pm 0.82$ & 0.0 \\
\hline & 250 & $23.33 \pm 1.03$ & 0.0 & 0.0 & 0.0 \\
\hline & 125 & 0.0 & 0.0 & 0.0 & 0.0 \\
\hline \multirow{4}{*}{ 07A } & 1000 & $24.50 \pm 0.84$ & $20.00 \pm 0.0$ & $24.50 \pm 0.55$ & $23.17 \pm 0.41$ \\
\hline & 500 & $21.83 \pm 1.17$ & $19.83 \pm 1.17$ & $22.83 \pm 0.75$ & $22.50 \pm 1.05$ \\
\hline & 250 & $20.50 \pm 1.22$ & $19.17 \pm 1.17$ & $20.67 \pm 1.21$ & $19.00 \pm 1.10$ \\
\hline & 125 & 0.0 & 0.0 & 0.0 & 0.0 \\
\hline \multirow{4}{*}{ 07B } & 1000 & $24.33 \pm 0.82$ & $20.17 \pm 0.75$ & $23.50 \pm 0.84$ & $22.17 \pm 0.75$ \\
\hline & 500 & $21.67 \pm 0.52$ & $19.67 \pm 1.03$ & $23.00 \pm 1.10$ & 0.0 \\
\hline & 250 & $20.17 \pm 0.75$ & $19.00 \pm 0.89$ & $20.50 \pm 0.84$ & 0.0 \\
\hline & 125 & 0.0 & 0.0 & 0.0 & 0.0 \\
\hline \multirow{4}{*}{ 08B } & 1000 & $24.33 \pm 0.52$ & $19.33 \pm 1.03$ & $21.50 \pm 0.84$ & $20.50 \pm 0.55$ \\
\hline & 500 & $22.17 \pm 0.75$ & $17.5 \pm 0.55$ & $18.67 \pm 0.82$ & $16.33 \pm 0.82$ \\
\hline & 250 & $21.33 \pm 1.03$ & $16.00 \pm 0.89$ & $15.53 \pm 0.55$ & $22.00 \pm 0.0$ \\
\hline & 125 & 0.0 & 0.0 & 0.0 & 0.0 \\
\hline \multirow[t]{4}{*}{ 09A } & 1000 & $25.17 \pm 0.41$ & $21.83 \pm 0.98$ & $25.00 \pm 0.89$ & $20.50 \pm 1.38$ \\
\hline & 500 & $23.50 \pm 0.55$ & $21.17 \pm 0.98$ & $24.17 \pm 0.75$ & $18.83 \pm 0.98$ \\
\hline & 250 & $22.17 \pm 0.75$ & $18.33 \pm 0.52$ & $21.50 \pm 1.38$ & $17.33 \pm 1.37$ \\
\hline & 125 & 0.0 & 0.0 & 0.0 & 0.0 \\
\hline \multicolumn{6}{|c|}{ FLUCLOXACILLIN } \\
\hline \multirow[t]{4}{*}{ FLMG01 } & 10000 & $23.17 \pm 0.41$ & $26.30 \pm 0.28$ & $22.83 \pm 0.59$ & $23.17 \pm 0.63$ \\
\hline & 5000 & $17.00 \pm 0.63$ & $20.00 \pm 0.22$ & $21.17 \pm 0.34$ & $23.83 \pm 0.51$ \\
\hline & 2500 & $17.00 \pm 0.89$ & $20.33 \pm 0.18$ & $20.83 \pm 0.45$ & $22.67 \pm 0.36$ \\
\hline & 1250 & 0.0 & 0.0 & 0.0 & 0.0 \\
\hline \multirow{4}{*}{ FLMG02 } & 10000 & $25.67 \pm 1.21$ & $21.33 \pm 1.03$ & $28.67 \pm 1.03$ & $19.83 \pm 0.98$ \\
\hline & 5000 & $22.50 \pm 1.38$ & $19.50 \pm 1.22$ & $27.67 \pm 1.21$ & $17.00 \pm 0.52$ \\
\hline & 2500 & $20.67 \pm 0.81$ & $18.50 \pm 0.55$ & $24.50 \pm 0.84$ & $16.00 \pm 0.0$ \\
\hline & 1250 & $17.67 \pm 1.37$ & $17.00 \pm 0.0$ & $19.00 \pm 0.69$ & $14.75 \pm 0.50$ \\
\hline \multirow{4}{*}{ FLMG03 } & 10000 & $31.67 \pm 0.82$ & $18.67 \pm 0.52$ & $29.50 \pm 1.22$ & 0.0 \\
\hline & 5000 & $29.50 \pm 0.55$ & $18.17 \pm 0.75$ & $27.83 \pm 0.98$ & 0.0 \\
\hline & 2500 & $28.33 \pm 0.82$ & $16.00 \pm 0.82$ & $27.67 \pm 0.82$ & 0.0 \\
\hline & 1250 & 0.0 & 0.0 & 0.0 & 0.0 \\
\hline \multirow{4}{*}{ FLLP04 } & 10000 & $30.67 \pm 1.10$ & $24.17 \pm 0.41$ & $30.50 \pm 0.55$ & 0.0 \\
\hline & 5000 & $27.33 \pm 0.52$ & $19.67 \pm 0.51$ & $27.83 \pm 0.41$ & 0.0 \\
\hline & 2500 & $26.83 \pm 0.41$ & 0.0 & $27.00 \pm 0.63$ & 0.0 \\
\hline & 1250 & 0.0 & 0.0 & 0.0 & 0.0 \\
\hline \multirow{4}{*}{ FLLP05 } & 10000 & $30.67 \pm 1.17$ & $24.00 \pm 0.63$ & $30.50 \pm 0.84$ & 0.0 \\
\hline & 5000 & $27.33 \pm 0.52$ & $19.67 \pm 1.03$ & $27.83 \pm 0.75$ & 0.0 \\
\hline & 2500 & $26.83 \pm 0.98$ & 0.0 & $27.00 \pm 0.63$ & 0.0 \\
\hline & 1250 & 0.0 & 0.0 & 0.0 & 0.0 \\
\hline \multirow{4}{*}{ FLLP06 } & 10000 & $26.17 \pm 0.41$ & $25.50 \pm 0.55$ & $24.00 \pm 0.63$ & $22.17 \pm 0.98$ \\
\hline & 5000 & $24.33 \pm 0.52$ & $23.00 \pm 0.63$ & $23.50 \pm 0.55$ & $21.17 \pm 0.75$ \\
\hline & 2500 & $22.67 \pm 0.52$ & $21.67 \pm 0.51$ & $21.00 \pm 0.08$ & $16.83 \pm 0.75$ \\
\hline & 1250 & 0.0 & 0.0 & 0.0 & 0.0 \\
\hline FLAR07 & 10000 & $24.00 \pm 0.50$ & $23.67 \pm 1.18$ & $25.50 \pm 0.68$ & $20.67 \pm 0.89$ \\
\hline & 5000 & $21.50 \pm 0.68$ & $18.00 \pm 0.53$ & $24.30 \pm 0.18$ & $24.00 \pm 0.0$ \\
\hline
\end{tabular}




\begin{tabular}{|c|c|c|c|c|c|}
\hline & 2500 & $17.83 \pm 1.00$ & $16.00 \pm 0.63$ & $23.30 \pm 0.18$ & $20.00 \pm 0.0$ \\
\hline & 1250 & 0.0 & 0.0 & 0.0 & 0.0 \\
\hline \multirow[t]{4}{*}{ FLAR08 } & 10000 & $20.00 \pm 0.89$ & $22.67 \pm 0.82$ & $25.83 \pm 0.41$ & 0.0 \\
\hline & 5000 & $18.50 \pm 0.55$ & $19.33 \pm 0.52$ & $22.50 \pm 1.05$ & 0.0 \\
\hline & 2500 & 0.0 & $16.33 \pm 0.07$ & 0.0 & 0.0 \\
\hline & 1250 & 0.0 & 0.0 & 0.0 & 0.0 \\
\hline \multicolumn{6}{|c|}{ CLOXACILLIN } \\
\hline \multirow{4}{*}{ CLLP01 } & 10000 & $29.83 \pm 0.41$ & $21.83 \pm 0.41$ & $29.50 \pm 0.84$ & $25.17 \pm 0.41$ \\
\hline & 5000 & $27.83 \pm 0.98$ & $19.33 \pm 0.52$ & $26.67 \pm 0.52$ & $25.33 \pm 0.82$ \\
\hline & 2500 & $26.17 \pm 0.40$ & $20.17 \pm 0.41$ & $25.67 \pm 0.52$ & $23.50 \pm 0.55$ \\
\hline & 1250 & 0.0 & 0.0 & 0.0 & 0.0 \\
\hline \multirow{4}{*}{ CLLP02 } & 10000 & $29.17 \pm 0.41$ & $22.00 \pm 1.26$ & $28.50 \pm 0.55$ & $29.50 \pm 0.55$ \\
\hline & 5000 & $27.50 \pm 0.84$ & $21.50 \pm 0.55$ & $28.33 \pm 0.52$ & $26.00 \pm 0.0$ \\
\hline & 2500 & $25.17 \pm 0.41$ & $20.83 \pm 0.75$ & $24.33 \pm 0.52$ & $25.67 \pm 0.51$ \\
\hline & 1250 & 0.0 & 0.0 & 0.0 & 0.0 \\
\hline \multirow{4}{*}{ CLLP03 } & 10000 & $29.00 \pm 0.89$ & $26.33 \pm 1.03$ & $27.33 \pm 0.82$ & $22.67 \pm 0.82$ \\
\hline & 5000 & $28.17 \pm 1.33$ & $23.67 \pm 0.82$ & $26.00 \pm 0.89$ & $16.67 \pm 0.52$ \\
\hline & 2500 & $26.33 \pm 1.03$ & $22.50 \pm 0.84$ & $24.00 \pm 0.89$ & $15.17 \pm 0.75$ \\
\hline & 1250 & 0.0 & 0.0 & 0.0 & 0.0 \\
\hline \multirow{4}{*}{ CLAR03 } & 10000 & $26.50 \pm 1.38$ & $14.33 \pm 1.37$ & $26.50 \pm 0.84$ & $18.50 \pm 1.38$ \\
\hline & 5000 & $23.50 \pm 1.0$ & 0.0 & $24.83 \pm 0.75$ & $14.83 \pm 0.41$ \\
\hline & 2500 & 0.0 & 0.0 & $23.67 \pm 0.82$ & $12.00 \pm 0.63$ \\
\hline & 1250 & 0.0 & 0.0 & 0.0 & 0.0 \\
\hline \multirow[t]{4}{*}{ CLAR04 } & 10000 & $26.17 \pm 0.98$ & $21.00 \pm 0.89$ & $24.17 \pm 1.17$ & $17.50 \pm 0.55$ \\
\hline & 5000 & $23.00 \pm 0.89$ & $23.17 \pm 0.75$ & $25.67 \pm 0.52$ & $11.50 \pm 0.55$ \\
\hline & 2500 & $23.33 \pm 0.52$ & $23.17 \pm 1.17$ & $20.30 \pm 0.52$ & 0.0 \\
\hline & 1250 & 0.0 & 0.0 & 0.0 & 0.0 \\
\hline \multirow{4}{*}{ CLMG } & 10000 & $27.50 \pm 0.84$ & $20.83 \pm 0.98$ & $23.67 \pm 0.82$ & $27.67 \pm 1.21$ \\
\hline & 5000 & $25.17 \pm 0.41$ & $25.33 \pm 0.52$ & $23.50 \pm 0.55$ & $24.83 \pm 0.41$ \\
\hline & 2500 & $23.17 \pm 0.41$ & $22.33 \pm 0.52$ & $22.50 \pm 0.55$ & $24.17 \pm 0.47$ \\
\hline & 1250 & 0.0 & 0.0 & 0.0 & 0.0 \\
\hline
\end{tabular}

$\mathrm{SEM}=$ standard error mean, Diameter of well $=10 \mathrm{~mm}$

Table 4: Antibacterial activity (mean zones of inhibition \pm SEM) of suspension samples.

\begin{tabular}{|c|c|c|c|c|c|}
\hline \multirow[b]{2}{*}{ Sample } & \multicolumn{5}{|c|}{ Organism } \\
\hline & $\begin{array}{c}\text { Concentration } \\
(\mu \mathrm{g} / \mathrm{mL})\end{array}$ & S. aureus & E. coli & B. subtilis & $\begin{array}{c}P . \\
\text { aeruginosa }\end{array}$ \\
\hline & & & AMOXICLLIN & & \\
\hline \multirow{4}{*}{ S01A } & 1000 & $21.83 \pm 1.22$ & $18.00 \pm 0.68$ & $22.50 \pm 0.81$ & 0.0 \\
\hline & 500 & $19.67 \pm 0.91$ & $15.83 \pm 0.31$ & $21.33 \pm 0.76$ & 0.0 \\
\hline & 250 & $18.67 \pm 0.91$ & $15.00 \pm 0.0$ & $18.83 \pm 0.42$ & 0.0 \\
\hline & 125 & 0.0 & 0.0 & 0.0 & 0.0 \\
\hline \multirow{4}{*}{$\mathrm{S} 02 \mathrm{~A}$} & 1000 & $14.00 \pm 0.22$ & $19.83 \pm 0.14$ & $16.17 \pm 0.14$ & $13.00 \pm 0.31$ \\
\hline & 500 & $15.50 \pm 0.19$ & $19.50 \pm 0.29$ & $15.00 \pm 0.22$ & $11.50 \pm 0.19$ \\
\hline & 250 & $13.50 \pm 0.19$ & $18.00 \pm 0.0$ & $13.33 \pm 0.18$ & 0.0 \\
\hline & 125 & 0.0 & 0.0 & 0.0 & 0.0 \\
\hline \multirow{4}{*}{ S02B } & 1000 & $19.33 \pm 0.18$ & $19.50 \pm 0.29$ & $19.83 \pm 0.26$ & 0.0 \\
\hline & 500 & $11.67 \pm 0.18$ & $16.83 \pm 0.26$ & $18.33 \pm 0.60$ & 0.0 \\
\hline & 250 & $15.17 \pm 0.14$ & $15.67 \pm 0.28$ & $15.38 \pm 0.34$ & 0.0 \\
\hline & 125 & 0.0 & 0.0 & 0.0 & 0.0 \\
\hline \multirow{4}{*}{ S02C } & 1000 & $18.33 \pm 0.17$ & $18.00 \pm 0.22$ & $18.17 \pm 0.14$ & 0.0 \\
\hline & 500 & $15.80 \pm 0.29$ & $16.33 \pm 0.18$ & $15.17 \pm 0.14$ & 0.0 \\
\hline & 250 & $12.50 \pm 0.19$ & $12.00 \pm 0.0$ & $12.17 \pm 0.14$ & 0.0 \\
\hline & 125 & 0.0 & 0.0 & 0.0 & 0.0 \\
\hline \multirow{4}{*}{ S03A } & 1000 & $20.00 \pm 0.0$ & $17.67 \pm 0.28$ & $20.33 \pm 0.56$ & $22.67 \pm 0.28$ \\
\hline & 500 & $18.67 \pm 0.28$ & $17.00 \pm 0.0$ & $18.50 \pm 0.57$ & $20.33 \pm 0.36$ \\
\hline & 250 & $19.17 \pm 0.45$ & $14.67 \pm 0.28$ & $18.00 \pm 0.38$ & $19.67 \pm 0.18$ \\
\hline & 125 & 0.0 & 0.0 & 0.0 & 0.0 \\
\hline \multirow{4}{*}{ S04A } & 1000 & $17.33 \pm 0.18$ & $16.83 \pm 0.14$ & $21.17 \pm 0.26$ & $18.33 \pm 0.18$ \\
\hline & 500 & $15.83 \pm 0.14$ & $14.67 \pm 0.17$ & $20.17 \pm 0.14$ & $17.33 \pm 0.28$ \\
\hline & 250 & $15.00 \pm 0.0$ & $13.00 \pm 0.0$ & $19.00 \pm 0.0$ & $14.67 \pm 0.18$ \\
\hline & 125 & 0.0 & 0.0 & 0.0 & 0.0 \\
\hline
\end{tabular}

\begin{tabular}{|c|c|c|c|c|c|}
\hline \multirow{4}{*}{$05 \mathrm{~A}$} & 1000 & $16.67 \pm 0.18$ & $24.83 \pm 0.26$ & $17.00 \pm 0.22$ & $26.33 \pm 0.18$ \\
\hline & 500 & $16.00 \pm 0.22$ & $23.00 \pm 0.53$ & $15.33 \pm 0.28$ & $24.50 \pm 0.19$ \\
\hline & 250 & $14.67 \pm 0.28$ & $22.33 \pm 0.78$ & $14.00 \pm 0.22$ & $21.67 \pm 0.18$ \\
\hline & 125 & 0.0 & 0.0 & 0.0 & 0.0 \\
\hline \multirow{4}{*}{ S06A } & 1000 & $20.00 \pm 0.00$ & $16.83 \pm 0.14$ & $17.83 \pm 0.14$ & $28.67 \pm 0.18$ \\
\hline & 500 & $16.33 \pm 0.18$ & $16.00 \pm 0.22$ & $14.67 \pm 0.18$ & $25.00 \pm 0.38$ \\
\hline & 250 & $14.00 \pm 0.00$ & $12.67 \pm 0.18$ & $12.67 \pm 0.18$ & $22.17 \pm 0.34$ \\
\hline & 125 & 0.0 & 0.0 & 0.0 & 0.0 \\
\hline \multirow{4}{*}{ S06B } & 1000 & $19.50 \pm 0.19$ & $20.67 \pm 0.18$ & $18.33 \pm 0.18$ & $20.62 \pm 0.18$ \\
\hline & 500 & $17.50 \pm 0.29$ & $20.50 \pm 0.29$ & $16.83 \pm 0.14$ & $17.00 \pm 0.00$ \\
\hline & 250 & $14.83 \pm 0.14$ & $21.67 \pm 0.36$ & $16.00 \pm 0.30$ & $16.83 \pm 0.45$ \\
\hline & 125 & 0.0 & 0.0 & 0.0 & 0.0 \\
\hline \multirow{4}{*}{ S06C } & 1000 & $19.83 \pm 0.14$ & $19.83 \pm 0.40$ & $15.17 \pm 0.34$ & $22.50 \pm 0.42$ \\
\hline & 500 & $15.50 \pm 0.19$ & $19.00 \pm 0.31$ & $14.50 \pm 0.19$ & $21.33 \pm 0.36$ \\
\hline & 250 & $16.67 \pm 0.18$ & $17.17 \pm 0.14$ & $13.83 \pm 0.14$ & $16.33 \pm 0.18$ \\
\hline & 125 & 0.0 & 0.0 & 0.0 & 0.0 \\
\hline \multirow{4}{*}{ S07A } & 1000 & $24.67 \pm 0.86$ & $19.67 \pm 0.36$ & $20.00 \pm 0.0$ & $13.17 \pm 0.40$ \\
\hline & 500 & $19.50 \pm 0.36$ & $19.0 \pm 0.22$ & $18.67 \pm 0.18$ & $17.17 \pm 0.63$ \\
\hline & 250 & $17.67 \pm 0.41$ & $18.33 \pm 0.52$ & $18.50 \pm 0.29$ & 0.0 \\
\hline & 125 & 0.0 & 0.0 & 0.0 & 0.0 \\
\hline \multirow{4}{*}{ S08A } & 1000 & $20.33 \pm 0.18$ & $19.33 \pm 0.18$ & $20.00 \pm 0.0$ & $24.67 \pm 0.36$ \\
\hline & 500 & $19.17 \pm 0.14$ & $18.16 \pm 0.14$ & $18.50 \pm 0.48$ & $22.50 \pm 0.19$ \\
\hline & 250 & $18.50 \pm 0.29$ & $16.00 \pm 0.22$ & $17.17 \pm 0.40$ & $20.33 \pm 0.18$ \\
\hline & 125 & 0.0 & 0.0 & 0.0 & 0.0 \\
\hline \multirow{4}{*}{ S08B } & 1000 & $22.00 \pm 0.00$ & $17.67 \pm 0.56$ & $20.17 \pm 0.14$ & $25.83 \pm 0.34$ \\
\hline & 500 & $20.33 \pm 0.18$ & $16.50 \pm 0.19$ & $18.67 \pm 0.35$ & $24.17 \pm 0.14$ \\
\hline & 250 & $19.67 \pm 0.18$ & $16.00 \pm 0.0$ & $17.17 \pm 0.14$ & $20.33 \pm 0.18$ \\
\hline & 125 & 0.0 & 0.0 & 0.0 & 0.0 \\
\hline \multicolumn{6}{|c|}{ FLUCLOXACILLIN } \\
\hline \multirow{4}{*}{ FLSMG01 } & 10000 & $27.17 \pm 0.41$ & $20.17 \pm 0.41$ & $32.83 \pm 0.75$ & $29.67 \pm 0.52$ \\
\hline & 5000 & $22.23 \pm 0.52$ & $18.00 \pm 0.63$ & $31.17 \pm 1.32$ & $28.83 \pm 0.41$ \\
\hline & 2500 & $11.17 \pm 0.41$ & 0.0 & $30.17 \pm 0.40$ & $27.83 \pm 0.41$ \\
\hline & 1250 & 0.0 & 0.0 & 0.0 & 0.0 \\
\hline \multirow[t]{4}{*}{ FLSMG02 } & 10000 & $25.50 \pm 0.55$ & $18.83 \pm 0.75$ & $25.60 \pm 1.05$ & $25.00 \pm 0.89$ \\
\hline & 5000 & $21.17 \pm 0.75$ & $17.67 \pm 0.82$ & $25.33 \pm 1.03$ & $25.5 \pm 0.55$ \\
\hline & 2500 & 0.0 & 0.0 & $25.00 \pm 0.63$ & $24.17 \pm 0.41$ \\
\hline & 1250 & 0.0 & 0.0 & 0.0 & 0.0 \\
\hline \multirow{4}{*}{ FLSMG03 } & 10000 & $24.67 \pm 1.03$ & $19.50 \pm 0.84$ & $27.33 \pm 0.51$ & $18.50 \pm 0.55$ \\
\hline & 5000 & $20.17 \pm 0.41$ & $15.50 \pm 0.55$ & $26.00 \pm 0.63$ & $15.67 \pm 0.52$ \\
\hline & 2500 & $14.83 \pm 0.98$ & 0.0 & $24.17 \pm 0.75$ & 0.0 \\
\hline & 1250 & 0.0 & 0.0 & 0.0 & 0.0 \\
\hline \multirow{4}{*}{ FLSLP04 } & 10000 & $34.67 \pm 0.52$ & $21.67 \pm 0.82$ & $30.50 \pm 0.55$ & $20.33 \pm 0.52$ \\
\hline & 5000 & $29.83 \pm 0.41$ & $18.50 \pm 0.55$ & $26.00 \pm 0.63$ & $16.67 \pm 0.52$ \\
\hline & 2500 & $29.33 \pm 0.52$ & 0.0 & $25.00 \pm 0.0$ & $11.00 \pm 0.0$ \\
\hline & 1250 & 0.0 & 0.0 & 0.0 & 0.0 \\
\hline \multirow{4}{*}{ FLSP05 } & 10000 & $30.17 \pm 0.41$ & $25.50 \pm 0.84$ & $37.83 \pm 0.75$ & $29.17 \pm 0.75$ \\
\hline & 5000 & $28.67 \pm 0.52$ & $24.50 \pm 0.84$ & $37.17 \pm 0.41$ & $25.33 \pm 1.03$ \\
\hline & 2500 & $28.0 .00 \pm 0.0$ & $20.33 \pm 0.51$ & $33.67 \pm 0.52$ & $20.67 \pm 0.81$ \\
\hline & 1250 & 0.0 & 0.0 & 0.0 & 0.0 \\
\hline \multirow{4}{*}{ FLSP06 } & 10000 & $32.33 \pm 0.41$ & $20.50 \pm 1.23$ & $30.33 \pm 0.52$ & $20.33 \pm 0.52$ \\
\hline & 5000 & $29.17 \pm 0.75$ & $17.83 \pm 0.98$ & $26.50 \pm 0.55$ & 0.0 \\
\hline & 2500 & $29.17 \pm 0.75$ & 0.0 & $25.00 \pm 0.63$ & 0.0 \\
\hline & 1250 & 0.0 & 0.0 & 0.0 & 0.0 \\
\hline \multirow{4}{*}{ FLSAR07 } & 10000 & $27.83 \pm 0.75$ & $20.83 \pm 0.41$ & $29.50 \pm 0.55$ & 0.0 \\
\hline & 5000 & $25.50 \pm 0.55$ & $16.33 \pm 0.52$ & $28.00 \pm 0.63$ & 0.0 \\
\hline & 2500 & $24.00 \pm 0.63$ & 0.0 & $26.33 \pm 0.52$ & 0.0 \\
\hline & 1250 & 0.0 & 0.0 & 0.0 & 0.0 \\
\hline & 10000 & $25.67 \pm 0.52$ & $28.83 \pm 0.75$ & $29.67 \pm 1.03$ & 0.0 \\
\hline FI CAPOR & 5000 & $24.17 \pm 0.98$ & $26.67 \pm 0.52$ & $27.50 \pm 1.05$ & 0.0 \\
\hline TLOATUO & 2500 & $21.83 \pm 0.41$ & $25.17 \pm 0.41$ & $25.50 \pm 0.55$ & 0.0 \\
\hline & 1250 & $19.83 \pm 0.75$ & $22.83 \pm 0.41$ & $24.50 \pm 1.22$ & 0.0 \\
\hline & & CLOX & ILLIN & & \\
\hline
\end{tabular}




\begin{tabular}{|c|c|c|c|c|c|}
\hline & 10000 & $33.83 \pm 0.41$ & $14.17 \pm 0.75$ & $20.67 \pm 0.82$ & $15.67 \pm 0.52$ \\
\cline { 2 - 6 } CLSLP01 & 5000 & $32.00 \pm 0.00$ & $11.17 \pm 0.41$ & $16.17 \pm 0.41$ & $14.33 \pm 0.52$ \\
\cline { 2 - 6 } & 2500 & $31.00 \pm 0.0$ & 0.0 & $11.00 \pm 0.0$ & 0.0 \\
\cline { 2 - 6 } & 1250 & 0.0 & 0.0 & 0.0 & 0.0 \\
\hline \multirow{4}{*}{ CLSLP02 } & 10000 & $20.33 \pm 0.52$ & $19.17 \pm 0.41$ & $20.33 \pm 0.52$ & $20.17 \pm 0.41$ \\
\cline { 2 - 6 } & 5000 & 0.0 & $14.83 \pm 0.41$ & $14.67 \pm 0.52$ & $12.00 \pm 0.00$ \\
\cline { 2 - 6 } & 2500 & 0.0 & 0.0 & 0.0 & 0.0 \\
\cline { 2 - 6 } & 1250 & 0.0 & 0.0 & 0.0 & 0.0 \\
\hline \multirow{4}{*}{ CLSLP03 } & 10000 & $19.83 \pm 0.41$ & $14.83 \pm 0.41$ & $30.17 \pm 0.41$ & $20.33 \pm 0.52$ \\
\cline { 2 - 6 } & 5000 & $16.17 \pm 0.41$ & $13.83 \pm 0.41$ & $26.00 \pm 0.0$ & $13.83 \pm 0.41$ \\
\cline { 2 - 6 } & 2500 & $14.67 \pm 0.52$ & 0.0 & $24.83 \pm 0.41$ & 0.0 \\
\cline { 2 - 6 } & 1250 & 0.0 & 0.0 & 0.0 & 0.0 \\
\hline \multirow{4}{*}{ CLMGS04 } & 10000 & $33.33 \pm 0.82$ & $20.83 \pm 0.98$ & $31.17 \pm 0.75$ & $20.00 \pm 0.63$ \\
\cline { 2 - 6 } & 5000 & $30.17 \pm 0.41$ & $11.17 \pm 0.41$ & $30.17 \pm 0.41$ & $14.83 \pm 0.75$ \\
\cline { 2 - 6 } & 2500 & $27.67 \pm 0.52$ & 0.0 & $29.50 \pm 0.55$ & 0.0 \\
\cline { 2 - 6 } & 1250 & 0.0 & 0.0 & 0.0 & 0.0 \\
\hline \multirow{4}{*}{ CLMG } & 10000 & $17.00 \pm 0.0$ & $21.17 \pm 0.75$ & $17.33 \pm 0.52$ & $24.00 \pm 0.63$ \\
\cline { 2 - 6 } & 5000 & $15.83 \pm 0.75$ & $23.00 \pm 0.0$ & $15.17 \pm 0.41$ & $20.50 \pm 0.55$ \\
\cline { 2 - 6 } & 2500 & $15.00 \pm 0.89$ & $22.50 \pm 0.58$ & $14.17 \pm 0.41$ & $22.00 \pm 0.0$ \\
\cline { 2 - 6 } & 1250 & 0.0 & 0.0 & 0.0 & 0.0 \\
\hline
\end{tabular}

$\mathrm{SEM}=$ standard error mean, Diameter of well $=10 \mathrm{~mm}$

Table 5: Antibacterial activity (mean zones of inhibition \pm SEM) of reference antibiotic samples.

\begin{tabular}{|l|l|l|l|l|}
\hline $\begin{array}{l}\text { Concentration }(\boldsymbol{\mu g} / \\
\mathrm{mL})\end{array}$ & S. aureus & E. coli & B. subtilis & P. aeruginosa \\
\hline AMOXICILLIN & & & & \\
\hline 5000 & $30.83 \pm 0.34$ & $27.00 \pm 0.0$ & $24.83 \pm 0.14$ & $24.50 \pm 0.89$ \\
\hline 2500 & $27.17 \pm 0.14$ & $24.67 \pm 0.28$ & $24.00 \pm 0.31$ & $21.67 \pm 0.18$ \\
\hline 1250 & $25.67 \pm 0.18$ & $21.67 \pm 0.47$ & $21.33 \pm 0.18$ & $20.00 \pm 0.0$ \\
\hline 625 & 0.0 & 0.0 & 0.0 & 0.0 \\
\hline FLUCLOXACILLIN & & & & \\
\hline 10000 & $35.17 \pm 0.14$ & $26.33 \pm 0.28$ & $38.00 \pm 0.31$ & $29.67 \pm 0.36$ \\
\hline 5000 & $31.50 \pm 0.89$ & $20.00 \pm 0.22$ & $35.17 \pm 0.14$ & $24.33 \pm 0.18$ \\
\hline 2500 & $29.67 \pm 0.18$ & $20.33 \pm 0.18$ & $32.83 \pm 0.14$ & $22.67 \pm 0.36$ \\
\hline 1250 & 0.0 & 0.0 & 0.0 & 0.0 \\
\hline CLOXACILLIN & & & & \\
\hline 10000 & $30.00 \pm 0.22$ & $23.67 \pm 0.34$ & $25.50 \pm 0.68$ & $26.00 \pm 0.22$ \\
\hline 5000 & $28.00 \pm 0.26$ & $18.00 \pm 0.53$ & $24.33 \pm 0.18$ & $28.33 \pm 0.28$ \\
\hline 2500 & $25.67 \pm 0.28$ & $19.67 \pm 0.36$ & $23.33 \pm 0.18$ & $25.67 \pm 0.36$ \\
\hline 1250 & 0.0 & 0.0 & 0.0 & 0.0 \\
\hline
\end{tabular}

SEM $=$ Standard error mean, diameter of well: $10 \mathrm{~mm}$

The internal standard yielded accurate results as increase or decrease in peak area of analyte also affected area of internal standard. Peak ratios were directly proportional to concentrations (Table 7).

\section{HPLC analysis of flucloxacillin and cloxacillin samples}

HPLC method was developed and validated for the evaluation of flucloxacillin and cloxacillin samples. Analysis was carried out in an ambient temperature $\left(25^{\circ} \mathrm{C}\right)$ with Shim pack CLC- $\mathrm{NH}_{2} \mathrm{C} 18$ column $150 \times$ $4.6 \mathrm{~mm}, 5$ microns column and a Finnigan Spectra System HPLC. A mobile phase consisting of acetonitrile: $0.01 \mathrm{M}$ potassium dihydrogen phosphate, $\mathrm{KH}_{2} \mathrm{PO}_{4}$, with a ratio of $60: 40(\mathrm{v} / \mathrm{v})$ yielded maximum sensitivity and separation with sample detection at UV wavelength of $225 \mathrm{~nm}$.

\section{HPLC analysis of reference flucloxacillin}

HPLC chromatograms of flucloxacillin as reference sample (Figure 5) and with acetaminophen (paracetamol) as an internal standard (Figure 6) were developed. The running time for the reference sample and the internal standard were within four (4) min. The peak at 3.146 $\mathrm{min}$ is for flucloxicillin whereas that for acetaminophen is $1.953 \mathrm{~min}$.
A four-point calibration curve was generated for flucloxacillin in the concentrations range of 25.35 to $152.10 \mu \mathrm{g} / \mathrm{mL}$ (Figure 7). The calibration curve provided a linear relationship between the area under curve $(y)$ and the concentrations of flucloxacillin with the regression equation of $\mathrm{y}=156.94 \mathrm{x}+0.0699\left(\mathrm{R}^{2}=0.995\right)$ (Figure 7). The residual points of the calibration curve were well distributed within acceptable limits (Figure 8).

The methods were validated using the International Conference on Harmonization guideline and the parameters therein. It was performed using a well-designed experiment and statistically relevant methods in accordance with International Conference on Harmonization (ICH) guidelines on validation of analytical procedures $[22,23]$. The linearity of the detector response for flucloxacillin was confirmed within 25.35 to $152.10 \mu \mathrm{g} / \mathrm{mL}$ (Figure 7).

Calibration curves were analyzed using a linear regression model

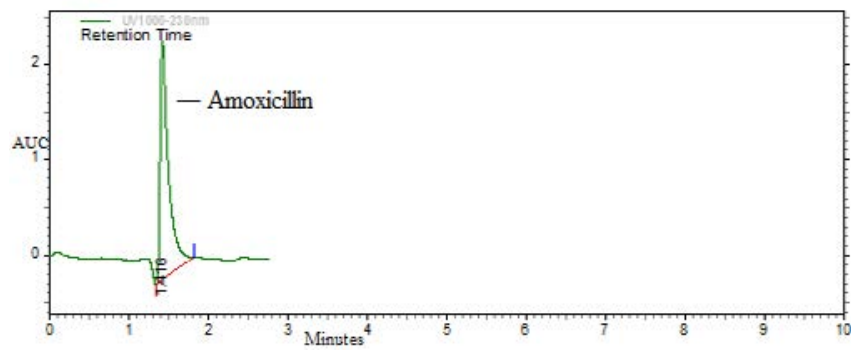

Figure 1: HPLC chromatogram of amoxicillin trihydrate as reference standard at wavelength $(\lambda) 230 \mathrm{~nm}$. AUC=Area under curve.

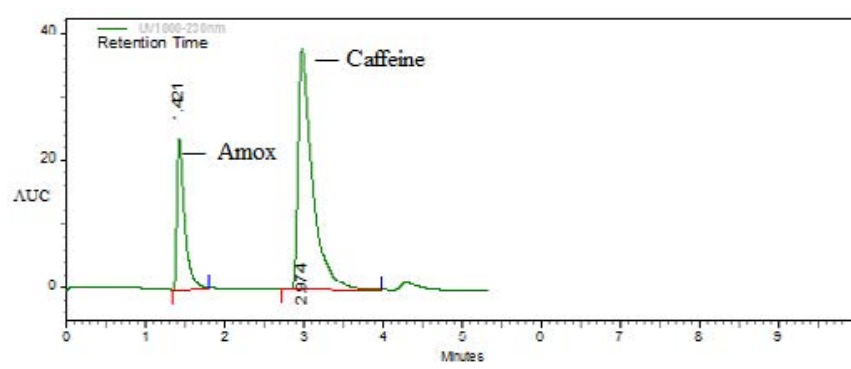

Figure 2: HPLC chromatogram of amoxicillin trihydrate as reference standard and caffeine anhydrous as internal standard at wavelength $(\lambda) 230 \mathrm{~nm}$. Amox: Amoxicllin.

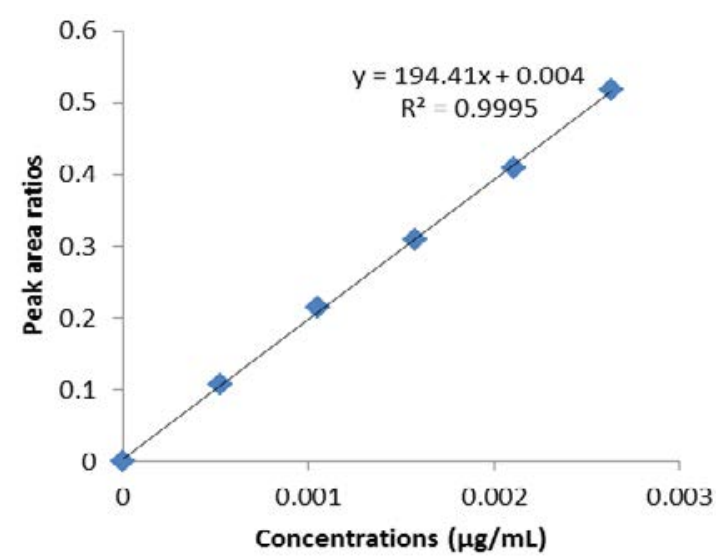

Figure 3: HPLC calibration curve of amoxicillin trihydrate (reference standard) 


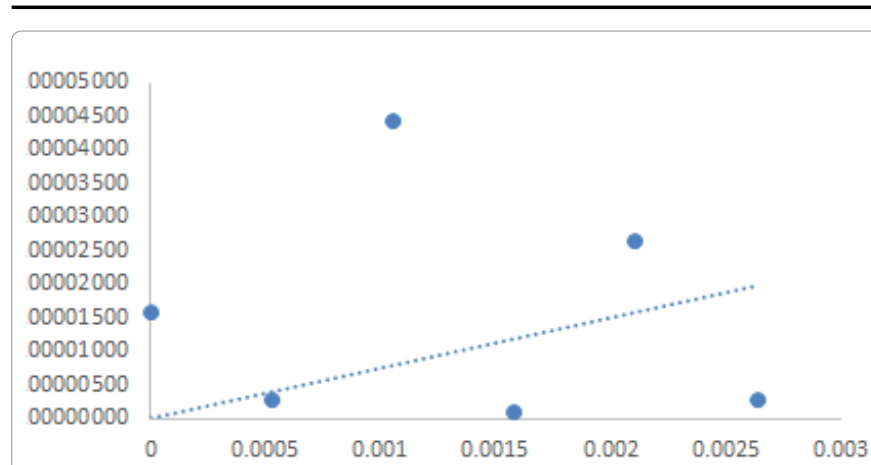

Figure 4: Residual plot of the HPLC calibration curve of amoxicillin trihydrate (reference standard).

\begin{tabular}{|c|c|}
\hline Parameter & Amoxicillin trihydrate \\
\hline Concentration range & 5.26 to $263.0 \mu \mathrm{g} / \mathrm{mL}$ \\
\hline Number & 5 \\
\hline Average values & 0.001315 \\
\hline Correlation coefficient & 0.9995 \\
\hline Relative standard deviation (\%) & 0.7483 \\
\hline Calibration equation & $\mathrm{y}=194.41 \mathrm{x}+0.004$ \\
\hline Limit of detection (LOD) & $1.6703 \times 10^{-5}$ \\
\hline Limit of quantification (LOQ) & $5.0617 \times 10^{-5}$ \\
\hline System suitability & 0.002 \\
\hline Method precision & $0.58 \%$ \\
\hline
\end{tabular}

LOD $=3.3 \times \sigma / S$, where $\sigma=S D E V$ of the responses, $S=$ slope of the regression line $L O Q=10 \times \sigma / S$, where $\sigma=S D E V$ of the responses, $S=$ slope of the regression line

Table 6: Statistical validation of the calibration data for quantitative determination of amoxicillin.

and linear co-efficients (Table 8). The limit of detection (LOD) and the limit of quantification (LOQ) were calculated using the signal-tonoise ratio ICH-Q2B, 1996] and were found to be $1.2837 \times 10^{-4}$ and 3.89 $\times 10^{-4} \mu \mathrm{g} / \mathrm{mL}[23]$.

Areas under curve ratios were directly proportional to concentrations as increase or decrease in peak area of analyte also affected area of internal standard (Table 9).

Accuracy for flucloxacillin was determined by the mean and SDV of the percentage recovery studies (Table 10).

\begin{tabular}{|c|c|c|}
\hline IS (AUC) & RS (AUC) & IS:RS (AUC ratio) \\
\hline 165429 & 478918 & 0.3454 \\
\hline 164384 & 472481 & 0.3478 \\
\hline 166733 & 479600 & 0.3477 \\
\hline 165828 & 474066 & 0.3498 \\
\hline 166732 & 474678 & 0.3513 \\
\hline 172047 & 493711 & 0.3484 \\
\hline & Mean=0.3484 \\
\hline & SDEV=0.00201 \\
\%RSD $=0.58 \%$ & \\
\hline
\end{tabular}

IS = Internal Standard, RS= Reference Standard, AUC= Area under curve, SDEV= Standard deviation, \%RSD = Percent relative standard deviation

Table 7: Analysis of homogenous reference amoxicillin solution for system suitability and precision analysis.

\section{HPLC analysis of cloxacillin}

HPLC chromatograms of cloxacillin as reference sample (Figure 9) and acetaminophen (paracetamol BP) as internal standard (Figure 10). The cloxacillin peak is at $2.874 \mathrm{~min}$ and that of acetaminophen is $1.933 \mathrm{~min}$.

A four-point calibration curve was generated for cloxacillin in the concentration range of 11.72 to $58.6 \mu \mathrm{g} / \mathrm{mL}$. The calibration curve provided a linear relationship between the peak area $(y)$ and the concentrations of amoxicillin injected $(\mathrm{x})$ with the regression equation of $y=787.78 x+0.0839\left(R^{2}=0.9986\right)$ (Figure 11). The residual points of the calibration curve were well distributed within acceptable limits (Figure 12).

The methods were validated using the International Conference on Harmonization guidelines and the parameters therein. It was performed using a well-designed experiment and statistically relevant methods in accordance with International Conference on Harmonization ( $\mathrm{ICH}$ ) guidelines on validation of analytical procedures (Q2A and Q2B). The linearity of the detector response for cloxacillin was from 11.72 to 58.6 $\mu \mathrm{g} / \mathrm{mL}$. The calibration curve (Figure 11) and the residuals (Figure 12) were inspected to asses linearity.

Calibration curves were analyzed using a linear regression model and linear coefficients (Table 11). The limit of detection (LOD) and the limit of quantification (LOQ) were calculated using the signal- tonoise ratio and were found to be $9.5246 \times 10^{-6} \mu \mathrm{g} / \mathrm{mL}$ and $2.8861 \times 10^{-5}$ $\mu \mathrm{g} / \mathrm{mL}$ respectively.

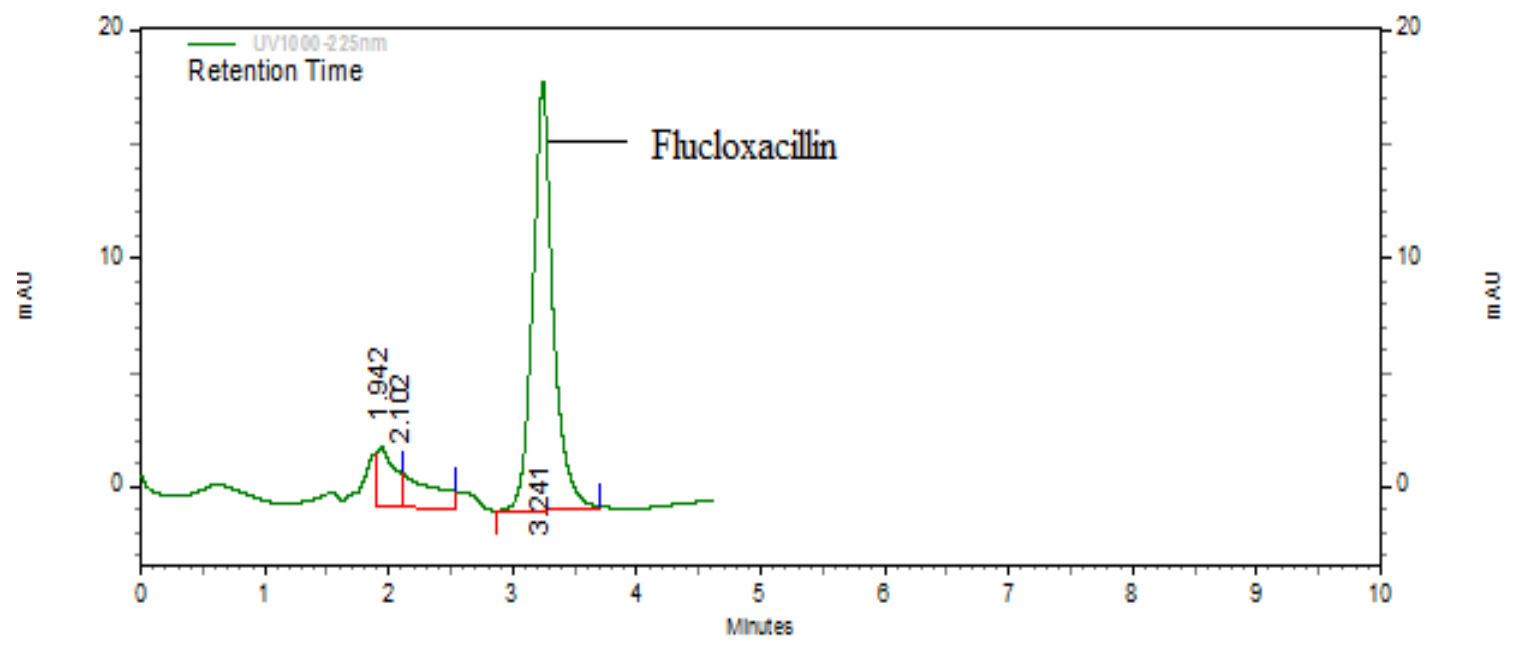

Figure 5: HPLC chromatogram of flucloxacillin as reference at wavelength $(\lambda)$ of $225 \mathrm{~nm}$. 


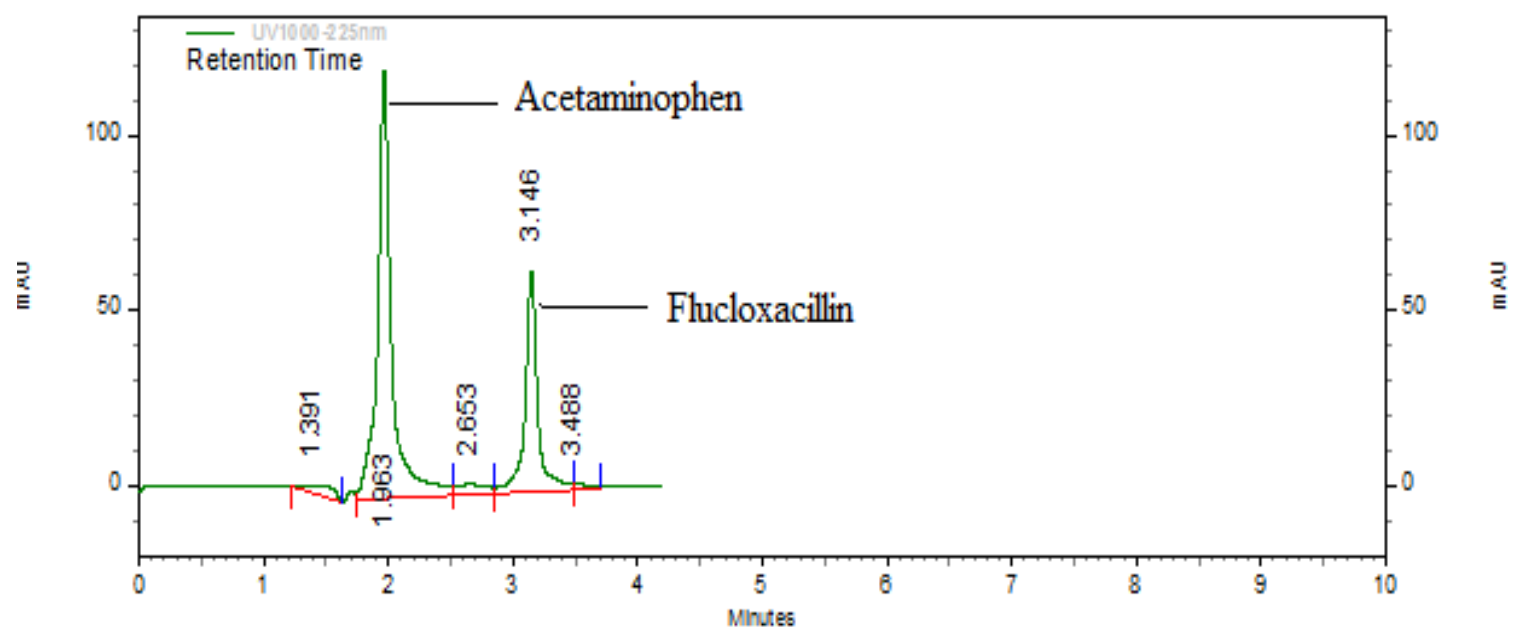

Figure 6: HPLC chromatogram of flucloxacillin as reference sample and acetaminophen as internal standard at wavelength $(\lambda) 225 \mathrm{~nm}$.

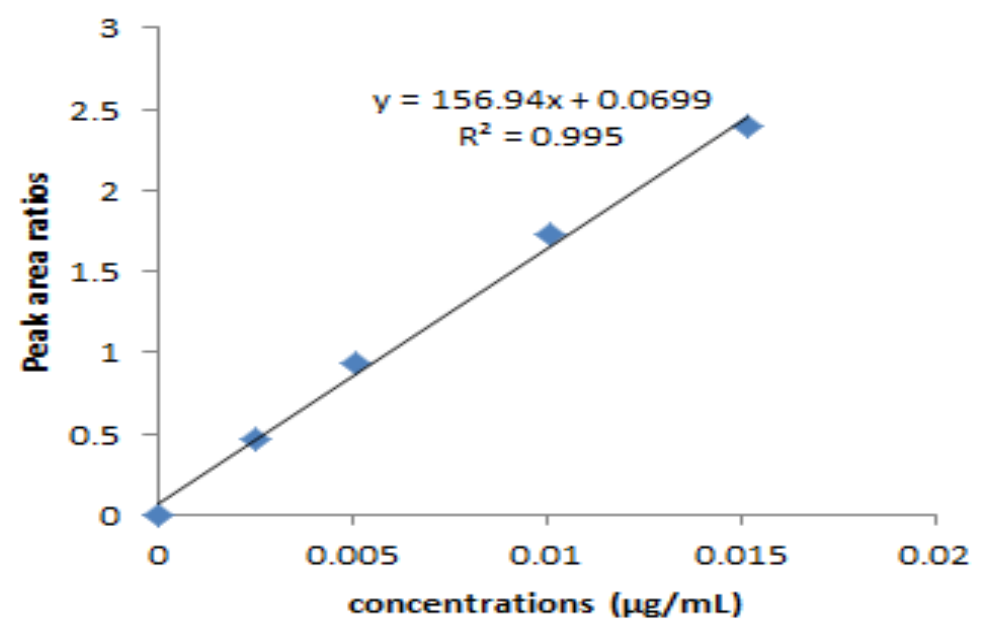

Figure 7: HPLC calibration curve of flucloxacillin (reference standard).

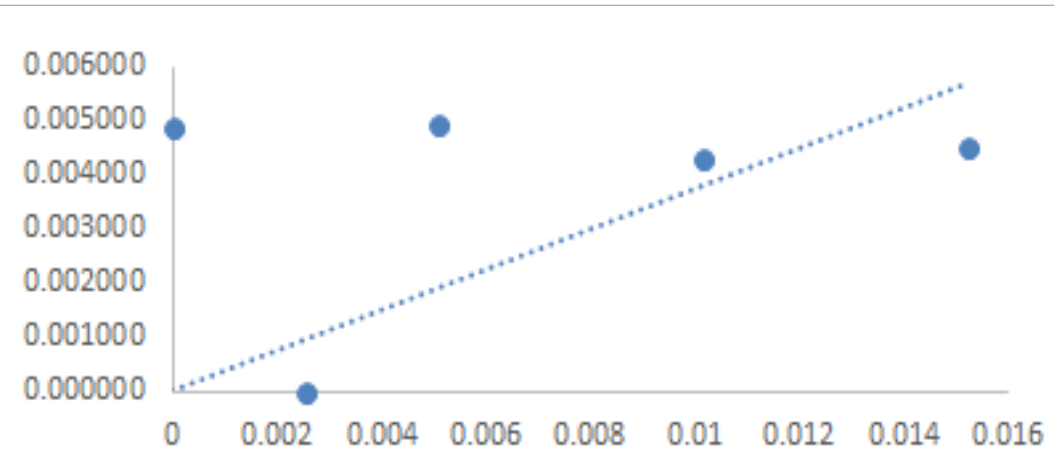

Figure 8: Residual plot of the HPLC calibration curve of flucloxacillin.

Peak ratios were directly proportional to concentrations as increase or decrease in peak area of analyte also affected area of internal standard (Table 12).

Accuracy for cloxacillin was determined by the mean and SDEV of the percentage recovery studies (Table 13).
HPLC analysis show that $75 \%$ amoxicillin capsules and $92.3 \%$ of suspension were within USP specification of 93.2 to $104.3 \%$ and 81.0 to $104.1 \%$ respectively. Sample of flucloxacillin capsules had $62.5 \%$ of the samples within specification of 96 to $120.5 \%$. All suspension samples were below the required USP specification. None of cloxacillin capsule samples were within the USP specification. All the suspension samples, 


\begin{tabular}{|c|c|}
\hline Parameter & Flucloxacillin \\
\hline Concentration range & $25.35-152.10 \mu \mathrm{g} / \mathrm{mL}$ \\
\hline Number & 4 \\
\hline Average values & 0.0066 \\
\hline Correlation coefficient $\left(\mathrm{R}^{2}\right)$ & 0.995 \\
\hline Relative standard deviation $(\%)$ & 0.9262 \\
\hline Calibration equation & $\mathrm{y}=156.94 \mathrm{x}+0.0699$ \\
\hline Limit of Detection & $1.2837 \times 10^{-4} \mathrm{\mu g} / \mathrm{mL}$ \\
\hline Limit of Quantification & $3.89 \times 10^{-4} \mu \mathrm{g} / \mathrm{mL}$ \\
\hline System suitability & 0.00253 \\
\hline Method precision & $0.25 \%$ \\
\hline
\end{tabular}

LOD $=$ Limit of detection, LOQ = Limit of quantification

$\mathrm{LOD}=3.3 \times \sigma / \mathrm{S}$, where $\sigma=\mathrm{SDEV}$ of the responses, $\mathrm{S}=$ slope of the regression line $L O Q=10 \times \sigma / S$, where $\sigma=S D E V$ of the responses, $S=$ slope of the regression line

Table 8: Statistical validation of the calibration data for quantitative determination of flucloxacillin.

\begin{tabular}{|c|c|c|}
\hline IS (AUC) & RS (AUC) & IS:RS (AUC ratio) \\
\hline 780955 & 799289 & 1.0235 \\
\hline 812336 & 830814 & 1.0227 \\
\hline 801131 & 823499 & 1.0279 \\
\hline 822182 & 843224 & 1.0256 \\
\hline 797503 & 814643 & 1.0215 \\
\hline & & Mean $=1.02424$ \\
& & SDEV $=0.00253$ \\
$\%$ RSD $=0.25 \%$
\end{tabular}

AUC $=$ Area under curve, IS = Internal standard, RS = Reference standard, SDEV= Standard deviation, \%RSD $=$ Percent relative standard deviation

Table 9: System suitability and precision parameters for reference flucloxacillin.

\begin{tabular}{|c|c|}
\hline Number $(\mathbf{n})$ & \% Recovery \\
\hline 1 & 92.36 \\
\hline 2 & 99.02 \\
\hline 3 & 107.87 \\
\hline 4 & 94.71 \\
\hline Mean & 98.49 \\
\hline SDEV & 6.834486 \\
\hline
\end{tabular}

$\mathrm{SDEV}=$ Standard deviation, $\mathrm{n}=4$

Table 10: Standard and internal standard recovery studies of reference flucloxacillin.

however, were within USP specification of 114.4 to $120.0 \%$. The USP specifications for amoxicillin trihydrate and flucloxacillin are 92.5 to $110 \%$ and 80 to $120 \%$ of stated amount for capsules and suspensions, respectively. Cloxacillin samples had 90 to $120 \%$ of API for both capsules and suspensions.

\section{Discussion}

The samples of the three different penicillins evaluated varied slightly from the standard reference samples in the microbiological evaluation. Suspensions had lower MICs as compared to the capsule samples. All samples in general showed higher MIC compared to the reference standards. The developed and validated HPLC methods were suitable for the intended purpose. HPLC analysis of the samples showed some of the samples contained the right amount of active pharmaceutical ingredients as stated in the USP [24] and BP [25] but they had higher MICs against the test bacteria.

\section{Antibacterial activities of penicillin samples}

Most of the penicillin samples were active against all the organisms but the mean zones of inhibition varied with different bacteria and sample as well as different concentrations. The pattern of zones of inhibition were not consistent as, in some cases, lower concentrations of the same sample had bigger or same sizes of zones of inhibition as compared to higher concentrations. This could be attributed to the fact that the antibiotic had to diffuse through the solid medium and the more concentrated they are, the higher the viscosity, hence, less diffusion rate. Consequently, the micro-dilution method was selected and used in the determination of the MIC as the test organisms are in direct contact with the antibiotic [26].

Helegbe et al. [27] reported that some selected antibiotics were active against some bacteria and recommended further studies on a larger scale. The current study, however, revealed higher MIC for the samples and this may be due to insufficient amount in the penicillin samples analyzed. A typical example is the report by Rahman et al. [28] which showed that zones of inhibition of amoxicillin samples against selected bacteria at $100 \mu \mathrm{g} / \mathrm{mL}$ were $19.5 \mathrm{~mm}$ for $E$. coli, $15.3 \mathrm{~mm}$ for $B$. subtilis and $17.0 \mathrm{~mm}$ for $S$. aureus. The current study on the other hand had no zones of inhibition at concentration below $250 \mu \mathrm{g} / \mathrm{mL}$. The amoxicillin samples had MIC of 125,180 and $220 \mu \mathrm{g} / \mathrm{mL}$ against $E$. coli, $S$. aureus and $B$. subtilis respectively and the current study, amoxicillin had MICs of 200, 200 and $300 \mu \mathrm{g} / \mathrm{mL}$ against E. coli, S. aureus and $B$. subtilis respectively.

There are differences between the literature values and that obtained from this study, but samples showed some level of sensitivity towards the test bacteria. Generally, there were differences in the sensitivity of Gram-negative and Gram-positive bacteria which could be due to the composition of the cell wall of two types of bacteria [29-31].

Some samples exhibited variations in the MIC. The antibacterial activity and MIC of samples varied from bacteria to bacteria which

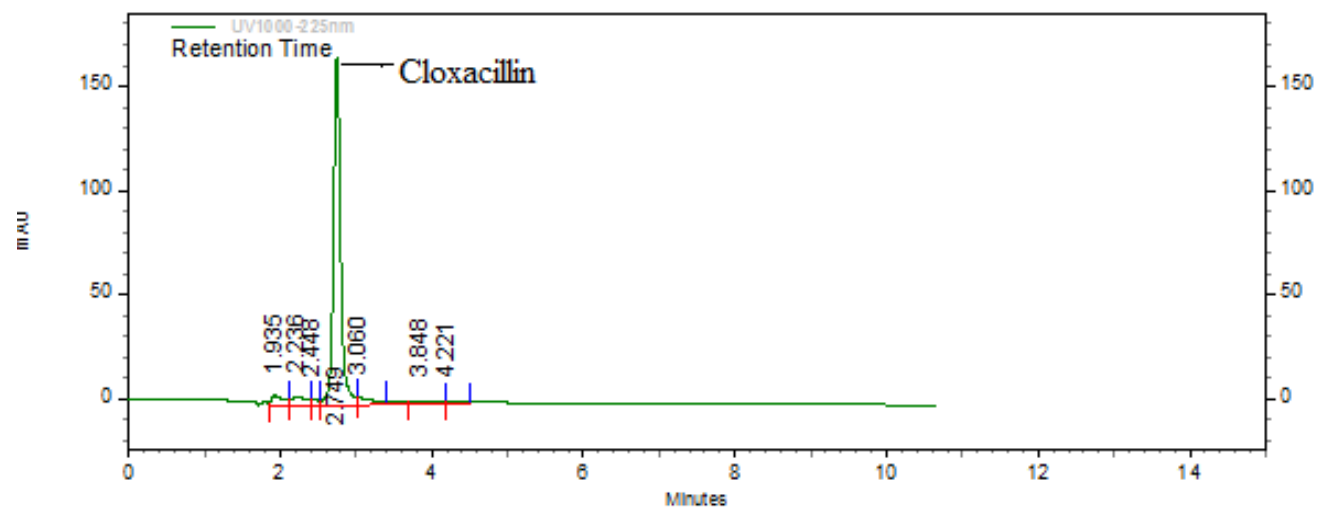

Figure 9: HPLC chromatogram of cloxacillin as reference at $\lambda 225 \mathrm{~nm}$. 


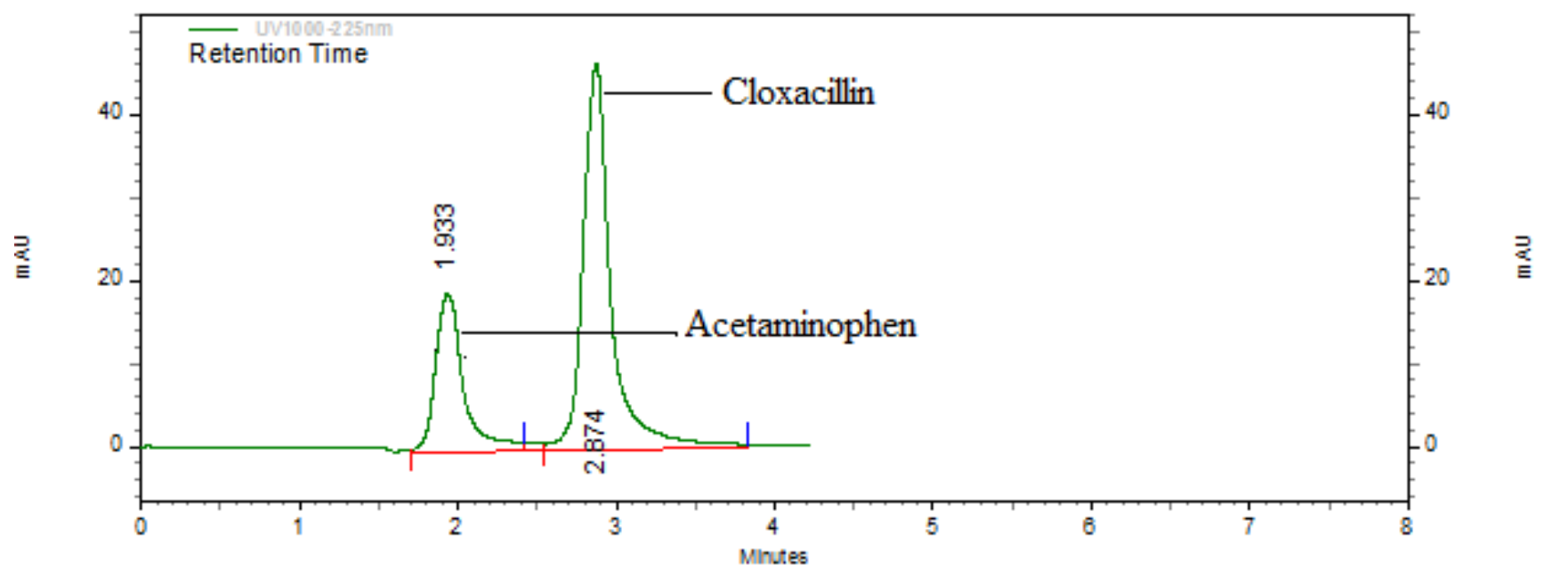

Figure 10: HPLC chromatogram of cloxacillin as reference and acetaminophen as internal standard at wavelength $225 \mathrm{~nm}$.

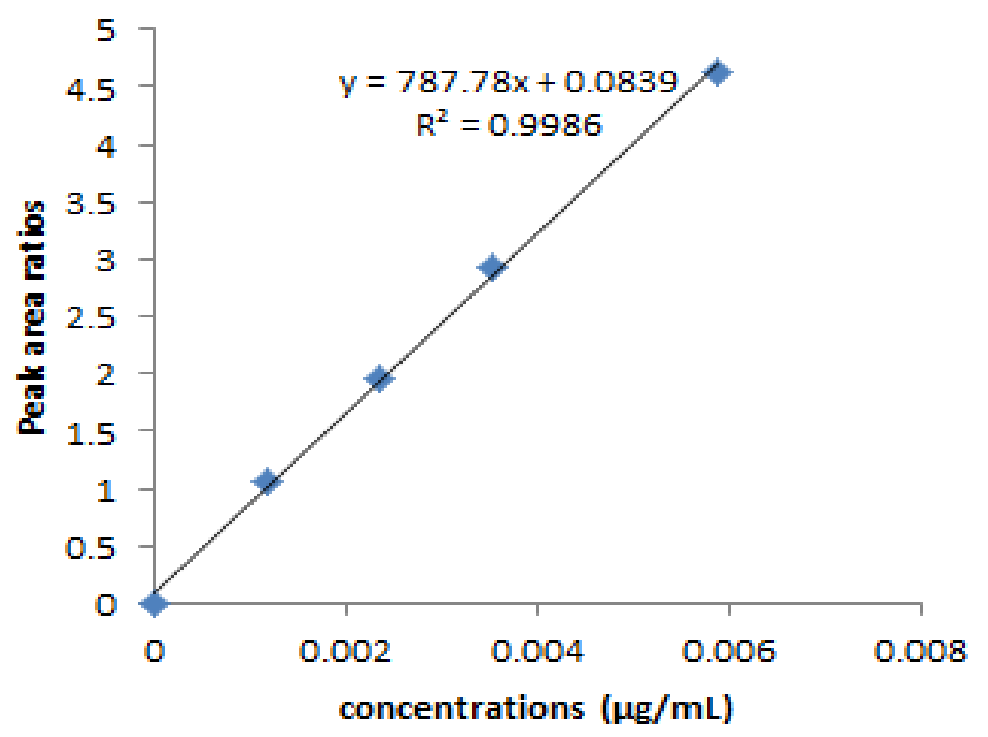

Figure 11: HPLC calibration curve of cloxacillin (reference standard).

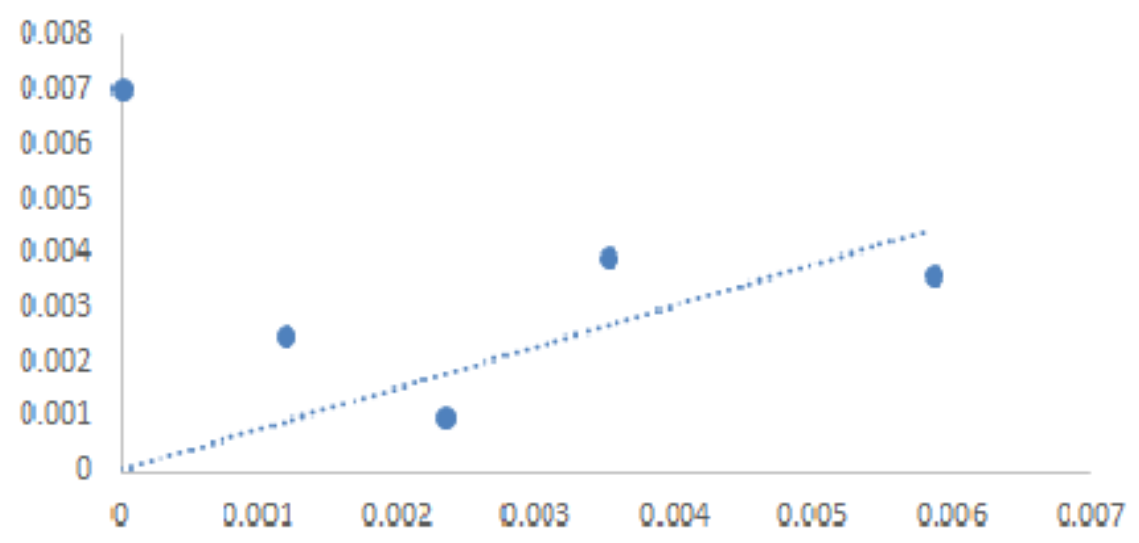

Figure 12: Residual plot of the HPLC calibration curve of cloxacillin (reference standard). 


\begin{tabular}{|c|c|}
\hline Parameter & Cloxacillin \\
\hline Concentration range & $\mu \mathrm{g} / \mathrm{mL}$ \\
\hline Number & 4 \\
\hline Average values & 0.0025784 \\
\hline Correlation coefficient & 0.9986 \\
\hline Relative standard deviation (\%) & 1.1340 \\
\hline Calibration equation & $\mathrm{y}=787.78 \mathrm{x}+0.0839$ \\
\hline Limit of detection (LOD) & $9.5246 \times 10^{-6} \mathrm{\mu g} / \mathrm{mL}$ \\
\hline Limit of quantification (LOQ) & $2.8861 \times 10^{-5} \mu \mathrm{g} / \mathrm{mL}$ \\
\hline System suitability & 0.00275 \\
\hline Method precision & $0.0336 \%$ \\
\hline
\end{tabular}

$\mathrm{LOD}=3.3 \times \sigma / \mathrm{S}$, where $\sigma=\mathrm{SDEV}$ of the responses, $\mathrm{S}=$ slope of the regression line $\mathrm{LOQ}=10 \times \sigma / \mathrm{S}$, where $\sigma=\mathrm{SDEV}$ of the responses, $\mathrm{S}=$ slope of the regression line

Table 11: Statistical validation of the calibration data for quantitative determination of reference cloxacillin.

\begin{tabular}{|c|c|c|}
\hline IS (AUC) & RS (AUC) & IS:RS (AUC ratio) \\
\hline 232461 & 195259 & 0.8391 \\
\hline 237534 & 200609 & 0.8391 \\
\hline 238890 & 185172 & 0.8445 \\
\hline 230526 & 187178 & 0.7751 \\
\hline 232653 & 190099 & 0.8171 \\
\hline Mean=0.81754, SDEV $=0.0275, \%$ RSD $=0.0336$ \\
\hline
\end{tabular}

$\mathrm{SDEV}=$ Standard deviation, $\% \mathrm{RSD}=$ Percent relative standard deviation, IS=Internal standard, AUC=Area under curve

Table 12: Internal standard, system suitability and precision parameters for reference cloxacillin.

\begin{tabular}{|c|c|}
\hline Number & \% Recovery \\
\hline 1 & 91.17 \\
\hline 2 & 91.51 \\
\hline 3 & 96.46 \\
\hline 4 & 113.41 \\
\hline Mean & 98.1375 \\
\hline SDV & 10.46475 \\
\hline SDEV= Standard deviation & \\
\hline
\end{tabular}

Table 13: Standard and internal standard recovery studies of reference cloxacillin $(n=4)$.

were similar to that of the reference sample. It was observed that, there were also variations among various brands and even batches within the same brand but variations were not significant $(\mathrm{p}>0.05)$.

Other reason that could account for differences in literature values and that of present study is the inoculum size of test organisms. Gbedema et al. [32] reported MIC of $0.46,640,0.29$ and $0.26 \mathrm{mg} /$ $\mathrm{mL}$ against E. coli, $P$. aeruginosa $S$. aureus and $B$. subtilis $10^{5} \mathrm{cfu} / \mathrm{mL}$ using the agar diffusion method. The inoculum size used in the present study was $10^{6} \mathrm{cfu} / \mathrm{mL}$ and it is higher than the inoculum size used by Gbedema et al. [32]. This might have resulted in the higher MICs recorded for the samples compared to the values reported by earlier workers $[28,32]$. Besides that, the micro-dilution method used in the determination of the MIC is reported to be a better approach than the agar diffusion technique [20,21].

Beta-lactams are inhibited by the beta lactamases produced by bacteria and the size of inoculum will have direct influence on the performance of the antibacterial agent. The inoculum size will determine the amount of beta-lactamase present to deactivate the beta lactam ring [33].

Comparison results from the biological and chemical method revealed that some of the samples passed the chemical assay but had higher MIC values. For this reason higher doses of these samples of amoxicillin are required for the treatment of infections due to these bacteria. Amoxicillin has enantiomers with its mirror image having the same chemical structure. A compound and its enantiomer show different activity with only one of its enantiomers usually biologically active [34].

Antibacterial activities of samples were similar but not the same as those of the reference standard. In general, flucloxacillin and cloxacillin samples were much active against $S$. aureus and $B$. subtilis compared to $E$. coli and $P$. aeruginosa. This could be due to the simple reason that isoxazolyl antibiotics are not very active against Gram-negative bacteria [27]. Samples in suspension forms showed higher activity as compared to the capsules against Gram-negative and Gram-positive bacteria. The possible reason could be due to the nature of formulation and the type of experimental design (In vitro) used. Capsules are to be swallowed and an acidic environment is required to enhance dissolution and release of API.

The isoxazolyl antibiotics such as flucloxacillin are not sensitive to penicillinase enzymes secreted by many penicillin-resistant bacteria, but able to bind to penicillin-binding proteins (PBPs) and inhibit peptidoglycan cross-linkage. This is made possible due to the presence of the isoxazolyl group on the side-chain of the penicillin nucleus which facilitates the $\beta$-lactamase resistance, since they are relatively intolerant of side-chain steric hindrance but it is not inactivated by $\beta$-lactamases. They are acid stable and have proven to be effective against $S$. aureus $[35,36]$.

There are some antibiotics that have been found to be substandard and counterfeited [37,38]. Substandard and counterfeit antibiotics are also noted to be one of the main causes of bacterial resistance to antibiotics [39]. Reports on substandard and/or counterfeit antibiotics on various markets have triggered investigations into their quality and activity. Different approaches, both biological and chemical analysis are used in the evaluations. The unavailability of specific materials such as the type of column and solvent systems to be used in chemical analysis in some laboratories in some developing countries and comparison of the results with specifications in standard reference books such as United State Pharmacopoeia (USP) and the British pharmacopoeia (BP) have made it necessary for the modification and validation of the existing methods with materials readily available to suit the type of analysis being performed especially in resource restrain areas or settings.

\section{HPLC analysis of penicillin samples}

The internal standard (IS), caffeine, was selected based on the fact that caffeine did not interact with the sample and absorbs at the same wavelength as the sample but it did not have the same retention time as the sample.

HPLC method with a good linearity depicts the direct proportionality between concentration of analytes and the area under curve of the peaks. With correlation coefficient (r) of 0.9997 and $\mathrm{R}^{2}$ of 0.9995 from the regression analysis of the calibration curve shows the direct proportional relationship between concentrations and peak area ratios. This represents an excellent linearity between them and how precise the HPLC method is. The method was shown to be linear. Observation of the calibration curve also confirms the linearity of the method developed (Figure 3).

The ability for the analyte of interest as far as this study is concerned, to elute in the presence of other compounds was ensured. A specific method is able to distinguish analyte even in the presence of other similar compounds. The ability of the amoxicillin to elute at the same retention time when spiked with the internal standard (Figure 
2) attests to the fact that the method was specific for the samples. The method can be used in the assessment of caffeine the analyte of interest. The internal standard was able to achieve the purpose for which it was intended (Table 12). Changes that could not be or difficult to control such as variations from run to run temperature and pressure during the run time were monitored by the internal standard. Relationship between the area under curve for the internal standard and area under curve for the reference standard yielded consistent area ratios (Table 13). The internal standard method is therefore considered the ideal as it yields accurate and precise results [40].

With respect to the suitability of a method, the USP [24] states that the percent relative standard deviation (\%RSD) from a six replicates runs of homogenous samples must not be more than 2 . The current method developed yielded RSD of $0.58 \%$ which is less than $2 \%$ and this is an indication of the suitability and precision of the method. The limits of detection and quantification values (Table 6) were indicative of how sensitive the method is. The attributes of the validation parameters considered shows that the method could be used to analyze amoxicillin samples within a considerable time using the readily available materials. The retention time of caffeine (internal standard) was 2.97 min whereas that of amoxicillin was 1.42 min at wavelength of $230 \mathrm{~nm}$ (Figure 2). The maximum absorption of the two compounds was detected at the same wavelength. Penicillins have no specific chromophore [41] and eluent must be maintained at wavelength less than $230 \mathrm{~nm}$ to obtain a meaningful detection limits. In this study, however amoxicillin was detected at wavelength of $230 \mathrm{~nm}$. The reason for the possible difference in retention time could be due to the different types of columns used and flow rates used. This was the method described by Ashnager and Naseri [42] to analyze amoxicillin samples at wavelength of $230 \mathrm{~nm}$ using Spherimage-80, ODS, 2-5 mm C18 column. A similar study of amoxicillin gave a retention time of 10 min for amoxicillin using the same buffer system and temperature whereas retention time of $1.42 \mathrm{~min}$ was recorded for amoxicillin in this current study. Abreu and Ortiz [43] also had a retention time of 5.2 min for amoxicillin using the C18 column at wavelength of $229 \mathrm{~nm}$ with mobile phase of phosphate buffer and acetonitrile. The limits of detection and quantification values as (Table 6) were indicative of how sensitive the method was. The specificity of the method was confirmed when the internal standard and reference standard were spiked with different concentrations of the same samples and they gave distinctive peaks of the two compounds at their respective retention times (Figure 2).

Analysis of the samples revealed that the content of all 16 different samples of the capsules were in the range of 81.53 to $104.34 \%$ (Tables 14 and 15). Twelve samples had their content within the USP [24] specification of 92.5 to $110.0 \%$. The sample with API of $93.2 \%$ was analyzed just 2 years before its expiry and few months after manufacturing and this means that the probability of the product failing later analysis before its expiry may be high.

The amount of API in suspension samples was $92.3 \%$ and these values are below the acceptable limit [24]. Percentages of active ingredient range of the suspension samples were from 81.03 to $104.1 \%$. Two batches were found to contain 81.0 and $81.33 \%$ active ingredient respectively and these samples have their API fall below the USP [24] specification. The fact that they were analyzed few months after their manufacture may indicate the samples may breakdown before expiry or did not contain the right amount of API. Almost $8 \%$ of the samples had their APIs below the USP [24] range.

After observing flow rates between 0.5 and $1 \mathrm{~mL} / \mathrm{min}$, the later was found to give an optimal signal-to-noise ratio with a reasonable separation and retention. In the quest of finding internal standard, various reference standards were used including amoxicillin cloxacillin and flucloxacillin. Injection of flucloxacillin and cloxacillin gave peaks with almost the same retention time and hence could not be used as the internal standard. Acetaminophen gave a retention time different from that of cloxacillin and flucloxacillin. Hence, it was used as internal standard for the analysis of cloxacillin and flucloxacillin samples. Environmental changes that could not be or difficult to control such as variations from run to run, temperature, pressure and power fluctuations during the run time were also monitored by the use of the internal standard in the analysis of the samples (Tables 9 and 12).

The limit of detection and limit of quantitative of the analysis indicate the sensitivity of the method. The direct proportional relationship between concentrations and peak area ratios with correlation coefficient $\mathrm{R}^{2}$ of 0.995 for flucloxacillin and 0.9986 for cloxacillin from the regression analysis of the calibration curves

\begin{tabular}{|c|c|c|c|c|c|c|c|c|}
\hline \multicolumn{9}{|c|}{ Sample / Amount / \% API } \\
\hline \multicolumn{3}{|c|}{92.5 to $110 \%$ (USP, 2011) } & \multicolumn{3}{|c|}{92.5 to $110 \%$ (USP, 2011) } & \multicolumn{3}{|c|}{$90-120 \%$ (USP, 2011) } \\
\hline \multicolumn{3}{|c|}{ Amoxicillin capsules $250 \mathrm{mg}$} & \multicolumn{3}{|c|}{ Flucloxacillin capsules $250 \mathrm{mg}$} & \multicolumn{3}{|c|}{ Cloxacillin capsules 250 mg } \\
\hline Sample code & Amount (mg) & $\%$ API & Sample code & Amount (mg) & $\%$ API & Sample code & Amount (mg) & $\%$ API \\
\hline $01 \mathrm{~A}$ & 260.85 & 104.34 & FLMG01 & 276.10 & 110.44 & CLLP01 & 156.00 & 62.40 \\
\hline 01B & 227.80 & 91.12 & FLMG02 & 161.63 & 64.65 & CLLP02 & 177.75 & 71.10 \\
\hline $02 \mathrm{~A}$ & 255.95 & 102.38 & FLMG03 & 111.85 & 44.74 & CLLP03 & 145.18 & 58.07 \\
\hline 02B & 244.83 & 97.93 & FLLP04 & 269.08 & 107.63 & CLAR04 & 139.60 & 55.84 \\
\hline $03 \mathrm{~A}$ & 203.83 & 81.53 & FLLP05 & 250.98 & 100.39 & CLAR05 & 201.95 & 80.78 \\
\hline 03B & 240.15 & 96.06 & FLLP06 & 239.90 & 95.96 & CLAR06 & & \\
\hline $03 \mathrm{C}$ & 244.53 & 97.81 & FLAR07 & 301.13 & 120.45 & CLMG & & \\
\hline $04 \mathrm{~A}$ & 230.07 & 92.03 & FLAR08 & 147.65 & 59.06 & & & \\
\hline 05A & 237.45 & 94.98 & & & & & & \\
\hline 06A & 217.20 & 86.88 & & & & & & \\
\hline 06B & 253.48 & 101.39 & & & & & & \\
\hline 06C & 238.58 & 95.43 & & & & & & \\
\hline 08A & 232.97 & 93.19 & & & & & & \\
\hline \multicolumn{9}{|c|}{ Amoxicillin capsules $500 \mathrm{mg}$} \\
\hline 07A & 480.00 & 96.00 & & & & & & \\
\hline 07B & 481.85 & 96.37 & & & & & & \\
\hline 09A & 493.15 & 98.63 & & & & & & \\
\hline
\end{tabular}

Table 14: HPLC analysis of amoxicillin, flucloxacillin and cloxacillin capsule samples. 


\begin{tabular}{|c|c|c|c|c|c|c|c|c|}
\hline \multicolumn{9}{|c|}{ Sample / Amount / \% API } \\
\hline \multicolumn{3}{|c|}{80 to $120 \%$ (USP, 2011) } & \multicolumn{3}{|c|}{80 to $120 \%$ (USP, 2011) } & \multicolumn{3}{|c|}{90 to $120 \%$ (USP, 2011) } \\
\hline \multicolumn{3}{|c|}{ Amoxicillin (125 mg/5 mL) } & \multicolumn{3}{|c|}{ Flucloxacillin (125 mg/5 mL) } & \multicolumn{3}{|c|}{ Cloxacillin (125 mg/5 mL) } \\
\hline Sample code & Amount & $\%$ API & Sample code & Amount & $\%$ API & Sample code & Amount & $\%$ API \\
\hline S01 & 117.56 & 94.05 & FLMG01 & 52.66 & 42.13 & CLSLP01 & 140.28 & 112.22 \\
\hline S02A & 101.29 & 81.03 & FLMG02 & 47.51 & 38.01 & CLSP02 & 149.96 & 119.97 \\
\hline S02B & 114.15 & 91.32 & FLMG03 & 48.03 & 38.42 & CLSLP03 & 143.05 & 114.44 \\
\hline S02C & 101.66 & 81.33 & FLLP04 & 48.91 & 39.13 & CLSMG04 & 143.86 & 115.09 \\
\hline S03A & 120.56 & 96.45 & FLLP05 & 58.21 & 46.57 & CLSMG05 & & \\
\hline S04A & 117.30 & 93.84 & FLLP06 & 62.59 & 50.07 & & & \\
\hline S05A & 98.38 & 78.70 & FLAR07 & 45.05 & 36.04 & & & \\
\hline S06A & 125.53 & 100.42 & FLAR08 & 45.35 & 36.28 & & & \\
\hline S06B & 126.75 & 101.40 & & & & & & \\
\hline S06C & 127.23 & 101.79 & & & & & & \\
\hline S07A & 130.14 & 104.11 & & & & & & \\
\hline S08A & 121.20 & 96.96 & & & & & & \\
\hline S08B & 110.53 & 88.42 & & & & & & \\
\hline
\end{tabular}

API: active pharmaceutical ingredient

Table 15: HPLC analysis of amoxicillin, flucloxacillin and cloxacillin suspension samples.

and these indicate the level of linearity. For five runs of the same homogenous reference solution (Tables 9 and 12) the suitability and precision of the method were in the acceptable limit as stated in USP [24] with SDEV of 0.0025 and \%RSD of 0.25 for flucloxacillin and standard deviation of 0.028 and \%RSD of 0.034 for cloxacillin. All these values were less than $2 \%$ in the USP [24].

The range of recovery for flucloxacillin and cloxacillin were 92.4 to $107.9 \%$ and 91.2 to $113.4 \%$ respectively with an average percentage recovery of $98.5 \%$ for flucloxacillin and $98.1 \%$ for cloxacillin. These represent a high level of accuracy of the methods.

In the evaluation of flucloxacillin samples (capsules) using the acceptance limit of 92.5 to $110 \%$ as stated in USP [24], 5 out of 8 samples evaluated were within the specification of USP [24] with percentage of 95.96 to 120.45 representing $62.5 \%$ of samples. The remaining samples had API of 44.7 to $64.7 \%$ which did not meet the specification in USP [24].

All the samples of flucloxacillin suspension analyzed were in the range of 36.0 to $50.1 \%$. These content are outside the USP [24] and BP [25] range of acceptance limit of 80 to $120 \%$. These low amounts of APIs may be due to insufficient active ingredients or poor storage conditions of the samples leading to the degradation of the API.

Antibiotics of this quality are threat to patients, the nation, and the world at large. Patients receiving such antibiotics would obviously not respond to minimum doses and would have to resort to higher doses. The activity of these antibiotic samples that failed the various evaluations may lead to antibiotic resistance in previously susceptible organisms.

Ensuring the quality, efficacy and safety of antibiotics would go a long way to prevent the problems associated with substandard and counterfeit antibiotics. The regulatory authorities that are mandated to regulate medicines must intensify their effort to monitor the quality and conditions of storage conditions of these antibiotics in especially developing countries.

\section{Conclusion}

All the penicillin samples (amoxicillin, flucloxacillin and cloxacillin) evaluated showed activity against test bacteria (E. coli, $P$. aeruginosa, $S$. aureus and $B$. subtilis). The level of activity and concentrations of penicillin samples gave different zones of inhibitions against these bacteria. Amoxicillin was observed to have broad spectrum activity showing activity against all bacteria used in the evaluation. Flucloxacillin and cloxacillin samples were observed to have higher activity against Gram-positive bacteria as compared to Gram-negative bacteria. P. aeruginosa was found to be most resistant bacteria to the penicillin samples. Suspension samples exhibited higher activity compared to capsule formulations. The MICs of 200 to 800 $\mu \mathrm{g} / \mathrm{mL}$ were recorded for amoxicillin samples whereas flucloxacillin and cloxacillin samples had MIC of 500 to $1900 \mu \mathrm{g} / \mathrm{mL}$. All samples of flucloxacillin suspensions and cloxacillin capsules had their API below the USP specification. Almost $83 \%$ of amoxicillin samples contained the right amount of API compared to $32.1 \%$ of flucloxacillin and $44.4 \%$ of cloxacillin samples having the right amount of API.

\section{Acknowledgments}

We are grateful to Mr. Samuel Bekoe and Mr. James Oppong Kyekyeku of Department of Pharmaceutical Chemistry, and Dr. Edmund Ekuadzi, Department of Pharmacognosy, and Mr. Newman Osafo, Department of Pharmacology, Faculty of Pharmacy and Pharmaceutical Sciences, Kwame Nkrumah University of Science and Technology, Kumasi, Ghana for their technical assistance.

\section{References}

1. Shakoor O, Taylor RB, Behrens RH (1997) Assessment of the incidence of substandard drugs in developing countries. Trop Med Int Health 2: 839-845.

2. Wondemagegnehu E (1999) Counterfeit and substandard drugs in Myanmar and Vietnam. World Health Organization, Geneva.

3. Caudron JM, Ford N, Henkens M, Macé C, Kiddle-Monroe R, et al. (2008) Substandard medicines in resource-poor settings: a problem that can no longer be ignored. Trop Med Int Health 13: 1062-1072.

4. WHO (2010) Assessment of Medicines Regulatory Systems in sub-Sarahan African Countries: An Overview of Findings from 26 Assessment ReportS (Geneva: WHO). P 48.

5. Gibson L (2004) Drug regulators study global treaty to tackle counterfeit drugs. BMJ 328: 486 .

6. Moore T, Lee D, Konduri N, Kasonde L (2012) Assuring the quality of essential medicines procured with donor funds. Health, Nutrition and Population (HNP) Discussion Paper. World Bank, Washington, DC

7. Vila J, Pal T (2010) Update on antibacterial resistance in low-income countries: Factors favoring the emergence of resistance. Open Infect Dis J 4: 38-54.

8. Kelesidis T, Kelesidis I, Rafailidis PI, Falagas ME (2007) Counterfeit or substandard antimicrobial drugs: a review of the scientific evidence. J Antimicrob Chemother 60: 214-236.

9. Yankus W (2006) Counterfeit Drugs: Coming to a Pharmacy near you. The American Council on Science and Health Inc. New York, 14. 
Citation: Boadu RF, Agyare C, Yiadom MA, Adu F, Boamah VE, et al. (2015) In vitro Activity and Evaluation of Quality of Some Selected Penicillins on the Ghanaian Market using Developed HPLC Methods. Med chem 5: 001-014. doi:10.4172/2161-0444.1000235

10. Aldhous $P(2005)$ Counterfeit pharmaceuticals: murder by medicine. Nature 434: 132-136.

11. Ahmad I, Beg AZ (2001) Antimicrobial and phytochemical studies on 45 Indian medicinal plants against multi-drug resistant human pathogens. J Ethnopharmacol 74: 113-123

12. Diaz JA, Silva E, Arias MJ, Garzón M (2011) Comparative in vitro study of the antimicrobial activities of different commercial antibiotic products of vancomycin. BMC Clin Pharmacol 11: 9.

13. Gallo GG, Lancini G, Parenti F (1995) Antibiotics-A multidisciplinary approach: Prenum Press, New York, 278.

14. Pankey GA, Sabath LD (2004) Clinical relevance of bacteriostatic versus bactericidal mechanisms of action in the treatment of Gram-positive bacterial infections. Clin Infect Dis 38: 864-870.

15. Lohsiriwat D, Chinswangwatanakul V, Lohsiriwat V, Leelaratsamee A (2009) Efficacy and safety of parenteral amoxycillin/ clavulanate for prevention of surgical site infection following abdominal surgery. J Med Assoc Thai 92: 11671170.

16. Spielholz C (2011) Efficacy of a synbiotic chewable tablet in the prevention of antibiotic-associated diarrhea. Health 3: 110-115.

17. McEwen SA, Fedorka-Cray PJ (2002) Antimicrobial use and resistance in animals. Clin Infect Dis 34 Suppl 3: S93-93S106.

18. Keyes K, Lee MD, Maurer JJ, Torrence M, Isaacson R (2008) Microbial food safety in animal agriculture: Current topics: Antibiotics: mode of action, mechanisms of resistance, and transfer. Wiley \& Sons, 45-56.

19. Walsh C (2003) Where will new antibiotics come from? Nat Rev Microbiol 1: 65-70.

20. Agyare C, Koffuor GA, Boamah VE, Adu F, Mensah KB, et al. (2012) Antimicrobial and Anti-Inflammatory Activities of Pterygota macrocarpa and Cola gigantea (Sterculiaceae). Evid Based Complement Alternat Med 2012: 902394.

21. Girish H, Satish S (2008) Antibacterial activity of important medicinal plants on human pathogenic bacteria-a comparative analysis. World Appl Sci J 5: 267-271.

22. ICH-Q2A (1995) Guideline for Industry: Text on validation of analytical procedures.

23. ICH-Q2B (1996). Guideline for industry, Q2B validation of analytical procedures: Methodology.

24. United States Pharmacopeia (2011) United States Pharmacopeia Convention Inc. Rockville, Maryland, USA

25. British Pharmacopoeia (2010) British Pharmacopoeia Commission Secretariat, London, United Kingdom

26. Agyare C, Koffuor GA, Boakye YD, Mensah KB (2013) Antimicrobial and antiinflammatory properties of Funtumia elastica. Pharm Biol 51: 418-425.

27. Helegbe G, Anyidoho LY, Gyang F (2009) Screening of the efficacy of some commonly used antibiotics in Ghana. Res J Microbiol 4: 214-221.
28. Rahman MU, Gul S, Odhano EA (2008) Antimicrobial activities of Ferula assafoetida oil against Gram positive and Gram negative bacteria. Am Eurasian J Agric Environ Sci 4: 203-206.

29. Butaye $P$, Devriese LA, Haesebrouck $F$ (2003) Antimicrobial growth promoters used in animal feed: effects of less well known antibiotics on gram-positive bacteria. Clin Microbiol Rev 16: 175-188.

30. Gupte S (2007) Review of Medical Microbiology: 2nd Edition, Jaypee Brothers Publishers, New Dehli, India, 316.

31. Mirghaed A, Yadollahi F (2013) Evaluation of the chemical composition and in vitro antimicrobial activity of Rosmarinus officinalis, Zataria multiflora, Anethum graveolens and Eucalyptus globulus against Streptococcus iniae; the cause of zoonotic disease in farmed fish. Iran J Fish Sci 12: 702-716.

32. Gbedema SY, Adu F, Bayor MT, Annan K, Boateng JS (2010) Enhancement of antibacterial activity of amoxicillin by some Ghanaian medicinal plant extracts. Int J Pharm Sci Res 1: 145-152.

33. Lancini G, Parenti F, Gallo GG (1995) Antibiotics-A multidisciplinary approach Springer, New York, 278.

34. Nandanwar HS, Hoondal GS, Vohra RM (2005) Enzymatic production of D-amino acids. In: Microbial Enzymes and Biotransformations. Methods in Biotechnology 17: 91-104.

35. Smith J, Hamilton-Miller J, Knox R (1962) Isoxazolyl penicillins and penicillinase. Nature 195: 1300 - 1301.

36. Sutherland R, Croydon EA, Rolinson GN (1970) Flucloxacillin, a new isoxazolyl penicillin, compared with oxacillin, cloxacillin, and dicloxacillin. Br Med J 4: 455460 .

37. Newton PN, Green MD, Fernández FM, Day NP, White NJ (2006) Counterfeit anti-infective drugs. Lancet Infect Dis 6: 602-613.

38. Reidenberg MM, Conner BA (2001) Counterfeit and substandard drugs. Clin Pharmacol Ther 69: 189-193.

39. Okeke IN, Lamikanra A, Edelman R (1999) Socioeconomic and behavioral factors leading to acquired bacterial resistance to antibiotics in developing countries. Emerg Infect Dis 5: 18-27.

40. Kavittha KY, Geetha G, Venkatnarayanan R (2012) Development and validation of liquid chromatographic methods for the estimation of drugs in multi-component dosage forms. Pharmacie Globale (IJCP) 3.

41. Foulstone M, Reading C (1982) Assay of amoxicillin and clavulanic acid, the components of Augmentin, in biological fluids with high-performance liquid chromatography. Antimicrob Agents Chemother 22: 753-762.

42. Ashnagar A, Naseri NG (2007) Analysis of three penicillin antibiotics (ampicillin, amoxicillin and cloxacillin) of several Iranian pharmaceutical companies by HPLC. J Chem 4: 536-545.

43. Pires de Abreu LR, Ortiz RM, de Castro SC, Pedrazzoli J Jr (2003) HPLC determination of amoxicillin comparative bioavailability in healthy volunteers after a single dose administration. J Pharm Pharm Sci 6: 223-230. 